\title{
Recellularized Native Kidney Scaffolds as a Novel Tool in Nephrotoxicity Screening ${ }^{\text {[S }}$
}

\author{
Michele Fedecostante, Koen G. C. Westphal, Michele F. Buono, Natalia Sanchez Romero, \\ Martijn J. Wilmer, Janis Kerkering, Pedro Miguel Baptista, Joost G. Hoenderop, \\ and Rosalinde Masereeuw
}

Division of Pharmacology, Utrecht Institute for Pharmaceutical Sciences, Utrecht, The Netherlands (M.F., K.G.C.W., M.F.B., N.S.R., R.M.); Aragon's Health Science Institutes (IACS), Zaragoza, Spain (N.S.M.); Departments of Pharmacology and Toxicology (M.J.W., J.K.) and Physiology (J.G.H.), Radboud Institute for Molecular Life Sciences, Radboud university medical center, Nijmegen, The Netherlands; Aragon Health Research Institute (IIS Aragon), Zaragoza, Spain (P.M.B.); Liver and Digestive Diseases Networking Biomedical Research Centre (CIBERehd), Madrid, Spain (P.M.B.); Jiménez Díaz Foundation Health Research Institute, Madrid, Spain (P.M.B.); and Department of Biomedical and Aerospace Engineering, Carlos III University of Madrid, Spain (P.M.B.)

Received January 31, 2018; accepted June 28, 2018

\begin{abstract}
Drug-induced kidney injury in medicinal compound development accounts for over $\mathbf{2 0} \%$ of clinical trial failures and involves damage to different nephron segments, mostly the proximal tubule. Yet, currently applied cell models fail to reliably predict nephrotoxicity; neither are such models easy to establish. Here, we developed a novel three-dimensional (3D) nephrotoxicity platform on the basis of decellularized rat kidney scaffolds (DS) recellularized with conditionally immortalized human renal proximal tubule epithelial cells overexpressing the organic anion transporter 1 (ciPTEC-OAT1). A 5-day SDS-based decellularization protocol was used to generate DS, of which $100-\mu \mathrm{m}$ slices were cut and used for cell seeding. After $\mathbf{8}$ days of culturing, recellularized scaffolds (RS) demonstrated 3D-tubule formation along with tubular epithelial
\end{abstract}

characteristics, including drug transporter function. Exposure of RS to cisplatin (CDDP), tenofovir (TFV), or cyclosporin A (CsA) as prototypical nephrotoxic drugs revealed concentration-dependent reduction in cell viability, as assessed by PrestoBlue and Live/Dead staining assays. This was most probably attributable to specific uptake of CDDP by the organic cation transporter 2 (OCT2), TFV through organic anion transporter 1 (OAT1), and CsA competing for P-glycoprotein-mediated efflux. Compared with 2D cultures, RS showed an increased sensitivity to cisplatin and tenofovir toxicity after 24-hour exposure (9 and $\mathbf{2 . 2}$ fold, respectively). In conclusion, we developed a physiologically relevant 3D nephrotoxicity screening platform that could be a novel tool in drug development.

\section{Introduction}

Drug-induced kidney injury is an important cause of failure in clinical trials during early drug development. It has been reported that $82 \%$ of novel entities failed at preclinical stage owing to safety concerns, with $8 \%$ the result of nephrotoxicity issues. Moreover, $52 \%$ of the studies failed in the clinical phase owing to safety, with $9 \%$ the result of renal issues (Cook et al., 2014). This underlines the need for improved screening systems that can identify nephrotoxicity before drugs reach the clinic. Nephrotoxicity may involve damage to different nephron segments, and the mechanism is strictly related

This research was funded by the Marie Curie ITN project: BIOART (Grant no. 316690, EU-FP7-PEOPLE-ITN-2012) and by Spain's Ministerio de Economía y Competitividad through a predoctoral fellowship (BES-2012-059562) and from Instituto de Salud Carlos III (project PI15/00563).

https://doi.org/10.1124/dmd.118.080721.

S This article has supplemental material available at dmd.aspetjournals.org. to the drug type. Both glomerular and tubular injuries are recognized targets of drug toxicity, and continuous exposure to such compounds may lead to development of chronic kidney disease (Awdishu and Mehta, 2017). In a healthy kidney, the proximal tubule is often a main target as this segment plays a crucial role in the renal handling of endo- and xenobiotics. Proximal tubular epithelial cells (PTEC) are equipped with a range of transporters that cooperate in basolateral uptake and luminal excretion of xenobiotics, as well as transporters and receptors mediating reabsorption of essential nutrients (Nigam, 2015; Liu et al., 2016). Drugs can compromise these processes through interactions.

As a prerequisite for drug registration, safety studies have to be performed in animals, but the clinical translation is often difficult as animals do not always reliably predict the human situation. Nephrotoxicity studies are also performed in two-dimensional (2D) cell cultures, but translation to the in vivo situation is often not possible here as well (Sanchez-Romero et al., 2016). A primary concern of in vitro toxicity studies is the spectrum of different kidney cells, experimental read-outs, drug selected, and drug

ABBREVIATIONS: AQP-1, Aquaporin 1; BCRP, breast cancer resistance protein; CDDP, cisplatin; CHO, Chinese hamster ovary; ciPTEC, conditionally immortalized proximal tubule epithelial cells; ciPTEC-OAT1, conditionally immortalized proximal tubule epithelial cells overexpressing OAT-1; Cldn-2, Claudin-2; CsA, cyclosporine A; 2D, two-dimensional; 3D, three-dimensional; DAPI, 4', 6-diamidino-2-phenylindole; DS, decellularized rat kidney scaffolds; ECM, extracellular matrix; HBSS, Hanks' buffered solution; MATE-1, multidrug and toxin extrusion protein 1; MATE-2K, multidrug and toxin extrusion protein 2K; MRP-4, multidrug resistance-associated protein 4; OAT1, organic anion transporter 1; OCT2, Organic cation transporter 2; PCR, polymerase chain reaction; P-gp, P-glycoprotein; Pipes, 1,4-piperazinediethanesulfonic acid; PTEC, proximal tubule epithelial cells; RS, recellularized kidney scaffolds; RT, room temperature; $\mathrm{TC}_{50}$, drug concentration leading to a $50 \%$ reduction in cell viability; TFV, tenofovir; ZO-1, zonula occludens 1; Zsq, zosuquidar. 
concentration used for such purpose (Huang et al., 2015; Wilmer et al., 2016).

In the past few years, researchers have shown an increased interest in developing three-dimensional (3D) microphysiologic systems that serve as a platform for ex vivo modeling of renal drug clearance and drug-induced kidney injury. These models can be used for preclinical screening of new drugs prior to initiation of clinical trials, thus improving the drug development processes (Davies, 2014; Weber et al., 2016; King et al., 2017).

The first step in developing a 3D microphysiologic system is selecting the matrix that allows 3D culturing. Decellularizing whole animal organs (e.g., rats and pigs) results in acellular scaffolds that retain their original 3D architecture and a preserved extracellular matrix (ECM) (Yu et al., 2014; Peloso et al., 2016; Fedecostante et al., 2017), offering the basis for generating new viable organs. These newly generated scaffolds can provide a promising alternative to synthetic ones, as the native ECM is expected to guide tissue development in a physiologically relevant manner.

Selecting the proper cell model is an important factor in establishing a nephrotoxicity platform. In this study, we used human conditionally immortalized proximal tubule epithelial cells (ciPTEC). This cell line has been extensively characterized previously in terms of epithelial phenotype, transporters gene, and protein expression and function (Wilmer et al., 2010; Schophuizen et al., 2013; Jansen et al., 2014; Caetano-Pinto et al., 2016; Nieskens et al., 2016). More recently, Nieskens et al. (2016) developed ciPTEC-OAT1, the cell model used in the current study that overexpresses organic anion transporter 1 (OAT1; SLC22A6), and demonstrated its role in tenofovir (TFV)-induced toxicity.

In the present study, we optimized and validated an SDS-based decellularization protocol using kidneys obtained from surplus rats, recellularized with ciPTEC, which were characterized for renal epithelial markers, including drug transporters. For further validation of the applicability in nephrotoxicity screenings, recellularized native kidney scaffolds were exposed to toxic drugs and compared with ciPTEC 2D cultures. Specifically, we focused on three drugs: cis-diamminedichloroplatinum (II) (cisplatin, CDDP), TFV, and cyclosporine A (CsA). The results obtained with these nephrotoxicants in our 3D screening platform show that this tool is potentially suitable as a novel alternative in drug development.

\section{Materials and Methods}

\section{Kidney Retrieval}

For the generation of decellularized rat kidney scaffolds (DS), 10 surplus controls, untreated, healthy, male Wistar rats, were obtained from the Central Animal Laboratory of Utrecht (GDL, Utrecht, The Netherlands) plus 10 surplus male Wistar rats from our own breeding stock at the Brain Center Rudolf Magnus (UMC Utrecht, The Netherlands). For the first group, heparin ( $5000 \mathrm{IU} / \mathrm{ml}, 0.25 \mathrm{ml} / \mathrm{kg}$; Leo Pharma, Ballerup, Denmark) and pentobarbital (1 ml/kg; MilliporeSigma, St. Louis, MO), used as anesthetics, were administered intraperitoneally. Rats were sacrificed via exsanguination. For the second group, heparin and anesthesia were not administered as we received already deceased animals, carbon dioxide having been used for euthanasia. In both groups, the abdominal cavity was opened and the kidneys, ureters, and surrounding vasculature were identified. Systemic vasculature was closed by suturing the vena cava and abdominal and caudal mesenteric arteries with silk sutures. Next, the renal arteries of both kidneys were isolated and a custom-made steel cannula was inserted and fixed with silk sutures. An incision in the lower renal vein was made and perfusion with heparin solution [10 IU/ml in Hanks' buffered solution (HBSS); Life Technologies/Thermo Fisher Scientific, Waltham, MA] was set at $2 \mathrm{ml} / \mathrm{min}$ for 15 minutes. The kidneys were dissected from fat and surrounding vasculature, after which the cannulated kidneys were extracted and frozen at $-20^{\circ} \mathrm{C}$ for later decellularization.

\section{Perfusion Decellularization}

For the decellularization process, the kidneys were thawed and connected to a perfusion system while hanging. Decellularization was performed at room temperature (RT) by introducing $1 \%$ (w/v) SDS dissolved in HBSS at a flow rate of $2 \mathrm{ml} / \mathrm{min}$ with a peristaltic pump (Ismatec, Zürich, Switzerland). Visible decellularization could be observed by a color change from red to translucent white, approximately after 21 hours. Subsequently, the kidneys were washed with HBSS for 30 minutes, followed by a treatment of 30 minutes with $1 \%(\mathrm{v} / \mathrm{v})$ Triton X-100 (MilliporeSigma) in HBSS to remove excess SDS. After that, the kidneys were washed with HBSS (on ice) containing $1 \%(w / v)$ penicillin/streptomycin (Life Technologies/Thermo Fisher Scientific), $50 \mathrm{mg} / 1$ gentamicin (Life Technologies/Thermo Fisher Scientific), and $2.5 \mu \mathrm{g} / \mathrm{ml}$ amphotericin B (MilliporeSigma) for 48 hours at a flow rate of $1 \mathrm{ml} / \mathrm{min}$. To remove potential immunogenic DNA, the kidneys were treated with a DNase buffer [DNase I, $0.0025 \%$ (w/v), $5 \mathrm{mM} \mathrm{CaCl}_{2}, 5 \mathrm{mM} \mathrm{MgCl}_{2}$ in HBSS; MilliporeSigma] for 5 hours at RT. A final wash with HBSS containing $1 \times$ penicillin/streptomycin, $50 \mathrm{mg} / \mathrm{l}$ gentamicin, and $2.5 \mu \mathrm{g} / \mathrm{ml}$ amphotericin B was performed for 24 hours to remove any excess nuclear material or detergent. Prior to recellularization, the scaffold was sterilized with $0.2 \%(\mathrm{v} / \mathrm{v})$ peracetic acid in $4 \%(\mathrm{v} / \mathrm{v})$ ethanol for 10 minutes and rinsed three times with sterile HBSS containing $1 \times$ penicillin/streptomycin, $50 \mathrm{mg} / \mathrm{l}$ gentamicin, $2.5 \mu \mathrm{g} / \mathrm{ml}$ amphotericin B under a laminar flow cabinet until a $\mathrm{pH}$ of 7.4 was reached (adjusted with $1 \mathrm{M}$ hydrochloric acid).

\section{Decellularized Scaffold Analysis}

DNA Isolation. Briefly, small pieces of the scaffold and cadaveric rat kidney were lysed in lysis buffer [Milli-Q water (MilliporeSigma, Burlington, MA), $1 \mathrm{M}$ Tris- $\mathrm{HCl}, \mathrm{pH} 8,0.5 \mathrm{M} \mathrm{Na}_{2}$-EDTA- $2 \mathrm{H}_{2} \mathrm{O}, 10 \%$ (w/v) SDS, $\left.5 \mathrm{M} \mathrm{NaCl}\right]$ with proteinase $\mathrm{K}(0.1 \mathrm{mg} / \mathrm{ml}$; Thermo Fisher Scientific $))$ for 24 hours at $55^{\circ} \mathrm{C}$ and with $600 \mathrm{rpm}$ on a thermomixer (Eppendorf, Hamburg, Germany). DNA was further washed and purified using ice-cold isopropanol, $70 \%(\mathrm{v} / \mathrm{v})$ ethanol, and several centrifugation steps. The DNA pellet was air-dried for 20 minutes and resuspended in $100 \mu \mathrm{l}$ Milli-Q water. DNA was quantified using a NanoDrop ND-1000 spectrophotometer (Thermo Fisher Scientific).

Histology. For histology and immunofluorescence, sagittal halves of the decellularized, recellularized, or cadaveric kidneys were put in cassettes and fixated in $10 \%(\mathrm{v} / \mathrm{v})$ formaldehyde for 24 hours. The fixed tissue was then embedded in paraffin according to standard procedure. Tissue sections of $4 \mu \mathrm{m}$ were cut on a microtome (Leica Microsystems, Wetzlar, Germany). The slides were deparaffinized in xylene for 30 minutes, with two changes each of $100 \%(\mathrm{v} / \mathrm{v}), 96 \%(\mathrm{v} / \mathrm{v})$, and $70 \%(\mathrm{v} / \mathrm{v})$ ethanol, respectively, for 3 minutes, and finally they were rehydrated in $1 \times$ phosphate buffered saline (PBS) $+1 \%(\mathrm{v} / \mathrm{v})$ Tween20 (MilliporeSigma). To confirm decellularization and preservation of tissue architecture, Masson trichrome staining was performed. This staining is a three-color staining protocol used to discriminate collagen fibers from muscular tissues on histologic slides and to determine ECM presence on the specimen. Moreover, the nucleus stains black; cytoplasm, erythrocytes, and muscle, red; collagens, blue; and elastin, purple.

Immunofluorescence. For immunofluorescence, $4-\mu \mathrm{m}$ paraffin embedded tissue sections were placed on SuperFrost Ultra Plus microscope slides (Thermo Fisher Scientific) and dried for 1 day. The next day, the slides were deparaffinized as indicated above and rehydrated in $1 \times \mathrm{PBS}+1 \%(\mathrm{v} / \mathrm{v})$ Tween 20 (MilliporeSigma). An antigen retrieval step was done using a microwaveheated citrate buffer ( $\mathrm{pH}$ 6.0) for 20 minutes on $98^{\circ} \mathrm{C}$. Subsequently, slides were cooled to RT. After the slides were washed with $1 \times$ PBS, the tissue was incubated in a blocking solution [2\% (v/v) fetal calf serum, $2 \%(\mathrm{w} / \mathrm{v})$ bovine serum albumin, and $0.1 \%(\mathrm{v} / \mathrm{v})$ Tween 20 in $1 \times \mathrm{HBSS}]$. Primary antibodies were added for 1 hour at RT. A dilution of 1:50 was used for all primary antibodies. The antibodies used, diluted in blocking buffer, were: goat antitype IV collagen (Southern Biotech, Birmingham, AL), rabbit anti-laminin (MilliporeSigma), and rabbit anti-fibronectin (Abcam, Cambridge, England). After a washing with $1 \times$ PBS, the secondary antibodies (donkey anti-goat IgG Alexa 488, goat anti-rabbit IgG Alexa 488; Life Technologies/Thermo Fisher Scientific) were added in a concentration of 1:200 and incubated for 30 minutes at RT. As second confirmation of successful decellularization, 4', 6-diamidino-2-phenylindole (DAPI; $300 \mathrm{nM}$; MilliporeSigma) was used as nuclei counterstain. The sections were observed with an Olympus FV1000 (Tokyo, Japan) confocal microscope.

SDS Quantification. To identify the leftover SDS in the scaffold, quantitative assay Stains-All Dye (MilliporeSigma) was used, according to a protocol described previously [30]. In short, tissue samples were lysed in a collagenase buffer $(1-2 \mathrm{mg} / \mathrm{ml}$ in $50 \mathrm{mM}$ TES, $0.36 \mathrm{mM}$ Calcium chloride, $\mathrm{pH} 7.4$ (Treweek, J.B. et al. 2015) for 48 hours at $37^{\circ} \mathrm{C}$ at $350 \mathrm{rpm}$ on a thermomixer. 
Supernatant of $1 \mu 1$ was mixed with $100 \mu 1$ of a Stains-All Dye working solution ( $1 \mathrm{mg}$ Stains-All Dye in $1 \mathrm{ml}$ of 50:50 $\mathrm{H}_{2} \mathrm{O} /$ isopropanol, $1 \mathrm{ml}$ formamide, and

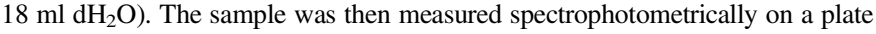
reader (Benchmark Plus; BioRad, Hercules, CA), with an absorbance of $438 \mathrm{~nm}$ ).

\section{Recellularized Scaffold Analysis}

Cell Culture of ciPTEC-OAT1. CiPTEC overexpressing the organic anion transporter 1 were cultured as described by Nieskens et al. (2016), in supplemented PTEC culture media as described by Jansen et al. (2014) and Nieskens et al. (2016). For the ciPTEC 2D model, cells were seeded at a density of 63,000 cells $/ \mathrm{cm}^{2}$, grown for 24 hours at $33^{\circ} \mathrm{C}$, and subsequently at $37^{\circ} \mathrm{C}$ for 7 days. ciPTEC required a temperature shift prior to each assay to mature and grow into fully differentiated epithelial cells forming a confluent cell monolayer.

Recellularization of Decellularized Native Kidney Scaffolds. Recellularization was performed on 150- $\mu \mathrm{m}$ thick slices of decellularized rat kidney scaffolds using Leica CM1950 cryostat (Leica Microsystems, Amsterdam, NL). Sequentially, the slices were sterilized for 10 minutes with $0.2 \%(\mathrm{v} / \mathrm{v})$ peracetic acid and rinsed three times with HBSS. For cell seeding, 500,000 cells were suspended in $30 \mu \mathrm{l}$ of medium and added on top of the scaffolds. The cell suspension was incubated for 2 hours at $33^{\circ} \mathrm{C}$, after which medium was added for 22 hours (also at $33^{\circ} \mathrm{C}$ ). Finally, the scaffolds were cultured in fresh medium for 1 week at $37^{\circ} \mathrm{C}$ (differentiation step).

Viability Assays. Cell passage numbers used for the nephrotoxicity studies were: for CDDP, 48, 50, and 52; for TFV, 51, 52, and 54; and for CsA, 51, 52, and 53. After the 8 days in culture, recellularized scaffolds and ciPTEC-OAT1 $2 \mathrm{D}$ cultures were treated with CDDP (24 hours, dissolved in complete medium), TFV [ 24 and 48 hours, dissolved in complete medium as reported by previously (Nieskens et al., 2016)], and CsA (24 hours dissolved in serum-free medium to get a proper drug solubility). Finally, a viability assay (PrestoBlue) was performed to investigate the nephrotoxic potential of the drugs.

PrestoBlue Assay. Recellularized scaffolds (RS) and cells grown in 96-well plates were cultured until day 7 and exposed to drugs or vehicle (control) for 24-48 hours, followed by a washout period of 1 hour before incubation with PrestoBlue (Thermo Fisher Scientific) 1:10 diluted with HBSS at $37^{\circ} \mathrm{C}$ for 1 hour. Samples were read with a fluorescence plate reader (Benchmark Plus; BioRad), with emission wavelength at $530 \mathrm{~nm}$ and excitation at $590 \mathrm{~nm}$. Background values were subtracted and normalized data were plotted.

Live/Dead Staining Assay. To further determine nephrotoxicity data for RS, matured samples after drug exposure were incubated with calcein AM $(2 \mu \mathrm{M}$; Thermo Fisher Scientific) and ethidium homodimer ( $2 \mu \mathrm{M}$; Thermo Fisher Scientific) diluted with $\mathrm{HBSS}$ at $37^{\circ} \mathrm{C}$ for 30 minutes. Imaging was performed with a Leica TCS SP8 confocal imaging system (Leica Microsystems). Images were analyzed with ImageJ version 5.1 and pixel intensities were obtained for each channel (green, calcein AM uptake; red, ethidium homodimer). Background values were subtracted and normalized ratios were plotted.

Immunofluorescence. For immunofluorescence, mature ciPTEC-OAT1 monolayers growing in WillCo-dishes (WillCo Wells B.V., Amsterdam, NL) and whole recellularized scaffolds were placed in 96-well plates and fixed with $4 \%(\mathrm{w} / \mathrm{v})$ Paraformaldehyde dissolved in PHEM buffer $1 \times(120 \mathrm{mM}$ Pipes, $50 \mathrm{mM}$ Hepes, $4 \mathrm{mM} \mathrm{MgCl}_{2}, 20 \mathrm{mM}$ EGTA) for 15 minutes. An antigen retrieval was done using a microwave-heated sodium-citrate buffer $(10 \mathrm{mM}, \mathrm{pH} 6.0$, adjusted with $\mathrm{HCl} 1 \mathrm{M})$ for 1 hour. Subsequently, the scaffolds were cooled down to RT. After a washing with $1 \times$ HBSS, the sample tissue was treated with a blocking solution [ $2 \%(\mathrm{v} / \mathrm{v})$ fetal calf serum, $3 \%(\mathrm{v} / \mathrm{v})$ bovine serum albumin (BSA), 3\% (v/v) normal goat serum, and $0.1 \%$ (v/v) Tween in HBSS]. The primary antibodies used, diluted in blocking buffer, were as follow: rabbit anti-human zonula occludens 1 (1:200; Invitrogen/Thermo Fisher Scientific) and claudin-2 (1:100; polyclonal rabbit anti-human; Thermo Fisher Scientific). The primary antibodies were incubated overnight at $4^{\circ} \mathrm{C}$. After a washing with $1 \times$ HBSS three times, the secondary antibody (goat anti-rabbit IgG Alexa 568; Life Technologies/Thermo Fisher Scientific), diluted in blocking buffer [0.1\% (w/v) BSA and $0.1 \%(\mathrm{v} / \mathrm{v})$ Tween-20 in HBSS], was added in a concentration of 1:200 and incubated for 1 hour at RT. Finally, DAPI anti-fade gold mounting medium (Invitrogen/Thermo Fisher Scientific) was used as nuclei counterstain to mount the scaffolds on WillCo-dishes. The samples were observed with a Leica TCS SP8 confocal imaging system (Leica Microsystems).

Gene Expression Analysis of Relevant Transporters. Briefly, mature ciPTEC-OAT1 cultured in six-well plates and RS $(N=20$ pooled per each RNA sample) were lysed, and RNA was extracted using RNA microfibrous kit
(RNeasy Mini Kit, Qiagen, Venlo, The Netherlands) according to the manufacturer's instructions and quantified using the NanoDrop ND-1000 spectrophotometer (Thermo Fisher Scientific). Reverse transcription of RNA to complementary DNA (cDNA) was performed using the iScript Reverse Transcription Supermix (Bio-Rad Laboratories) following manufacturer's instructions.

Quantitative Polymerase Chain Reaction (TaqMan). The mRNA expression of OAT1, organic cation transporter 2 (OCT2), P-glycoprotein (P-gp), breast cancer resistance protein (BCRP), multidrug resistance-associated protein 4 (MRP-4), aquaporin 1 (AQP-1), multidrug and toxin extrusion protein 1 (MATE-1), MATE$2 \mathrm{~K}$, organic anion transporter polypeptide $4 \mathrm{C} 1$ (OATP4C1), and glucose transporter 1 was examined in ciPTEC-OAT1 when cultured in flat monolayers and as recellularized kidney scaffolds. The polymerase chain reaction (PCR) reaction was performed according the TaqMan protocol manufacturer (Invitrogen/Thermo Fisher Scientific). Gene expression levels were normalized using CFX Manager software (Bio-Rad) to expression levels of the reference gene GAPDH and were expressed as fold increase compared with matured cells in well plates. Primer probe set sequences can be found in Supplemental Table 1.

Quantitative PCR (Syber Green). The mRNA expression of zonula occludens 1 (ZO-1) and claudin-2 (CLDN-2) was examined in ciPTEC-OAT1 when cultured in flat monolayers and as RS. The real-time PCR was performed using the iQ SYBR Green Supermix (Bio-Rad) as indicated in manufacturer's protocol and by means of CFX96 Real-Time PCR Detection System (Bio-Rad, Veenendaal, The Netherlands). The data were analyzed using CFX Manager Software version 3.1 (Bio-Rad) and expressed as fold increase compared with ciPTEC 2D model as the reference sample. HPRT1 was used as housekeeping gene for normalization. Specific sense and anti-sense primers for:

\section{HPRT1 (forward: ACATCTGGAGTCCTATTGACATCG; reverse: CCGCCCAAAGGGAACTGATAG), \\ zonula occludens 1 (forward: ATGGTGTCCTACCTAATTCAACTCAT; reverse: GCCAGCTACAAATATTCCAACATCA), and \\ claudin 2 (CLD-2) (forward: ACCTGCTACCGCCACTCTGT; reverse: CTCCCTGGCCTGCATTATCTC)}

were synthesized by Biolegio (Nijmegen, The Netherlands).

Live Imaging of Transport Activity. To determine transporter function in RS, live imaging was performed using specific fluorescent probes for transporters, viz. $\mathrm{ASP}^{+}$, fluorescein, and calcein-AM for OCT-2, OAT-1, and P-gp, respectively. $\mathrm{RS}$ and $\mathrm{ASP}^{+}(5 \mu \mathrm{m})$ in the presence or absence of cimetidine $(100 \mu \mathrm{M})$ and CDDP $(300 \mu \mathrm{M})$ were incubated for 10 minutes at $37^{\circ} \mathrm{C}$. RS and calcein-AM $(2 \mu \mathrm{M})$ alone or in the presence of zosuquidar (Zsq; $1 \mu \mathrm{M})$ or CsA $(1 \mathrm{mM})$ and Zsq $(1 \mu \mathrm{M})$ were incubated for 10 minutes at $37^{\circ} \mathrm{C}$. RS and fluorescein $(1 \mu \mathrm{M})$ in the presence or absence of probenecid $(100 \mu \mathrm{M})$, TFV $(500 \mu \mathrm{M})$, and the efflux pump inhibitors $(5 \mu \mathrm{M})$ were incubated for 5 minutes at $37^{\circ} \mathrm{C}$. Finally, DAPI anti-fade gold mounting medium (Invitrogen/Thermo Fisher Scientific) was used as nuclei counterstain to mount the scaffolds on WillCo-dishes. Samples were examined with a Leica TCS SP8 confocal imaging system (Leica Microsystems) and respective images of cellular uptake were obtained. From each single image three different regions in focus were selected and pixel intensities were determined using ImageJ version 5.1.

Data Analysis. All data are expressed as mean \pm S.D. of three independent experiments performed, at least, in duplicate. For the PrestoBlue viability assay, data were normalized for the untreated control (100\% viability) and the $\mathrm{TC}_{50}$ (the drug concentration leading to a $50 \%$ reduction in cell viability) was determined using the nonlinear fit " $\log$ (inhibitor) versus normalized response with variable slope" with GraphPad 6.07 from the percentage viability values. Statistical analysis was performed using one-way analysis of variance analysis followed by Dunnett's or Tukey's multiple comparison test, or, when appropriate, an unpaired Student's $t$ test with GraphPad Prism version 6.07 (La Jolla, CA). A $P$ value of $<0.05$ was considered significant.

\section{Results}

Morphologic Analysis of Decellularized Rat Native Kidney Scaffolds. The SDS-based (1\%) perfusion for 21 hours led to a visible decellularization of rat kidneys (Fedecostante et al., 2017). This step was followed by long wash steps, a short Triton $\times 100(1 \%)$ treatment to remove remaining SDS and a 5-hour DNase treatment to ensure a 
A

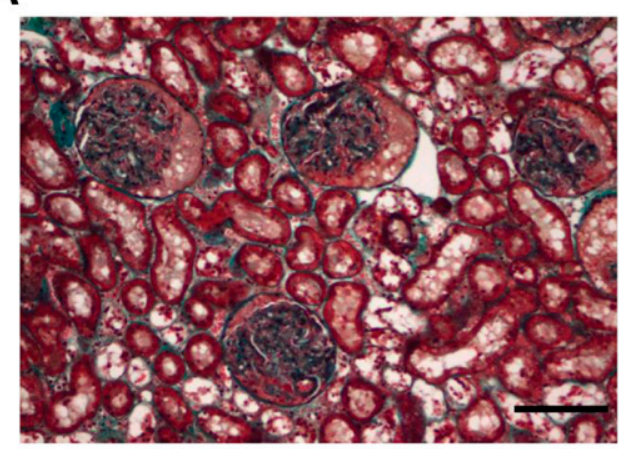

C

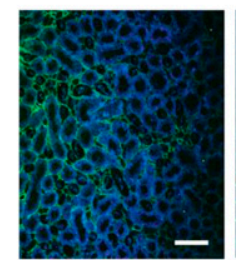

G

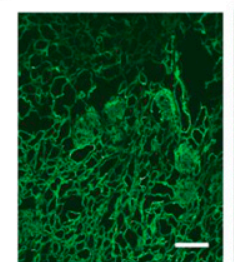

$\mathbf{L}$

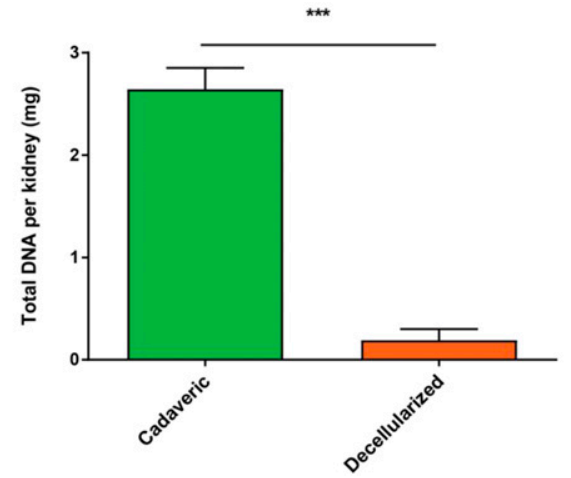

E

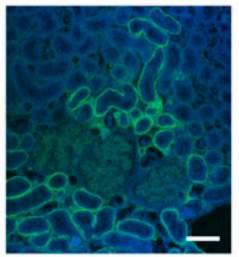

H
B
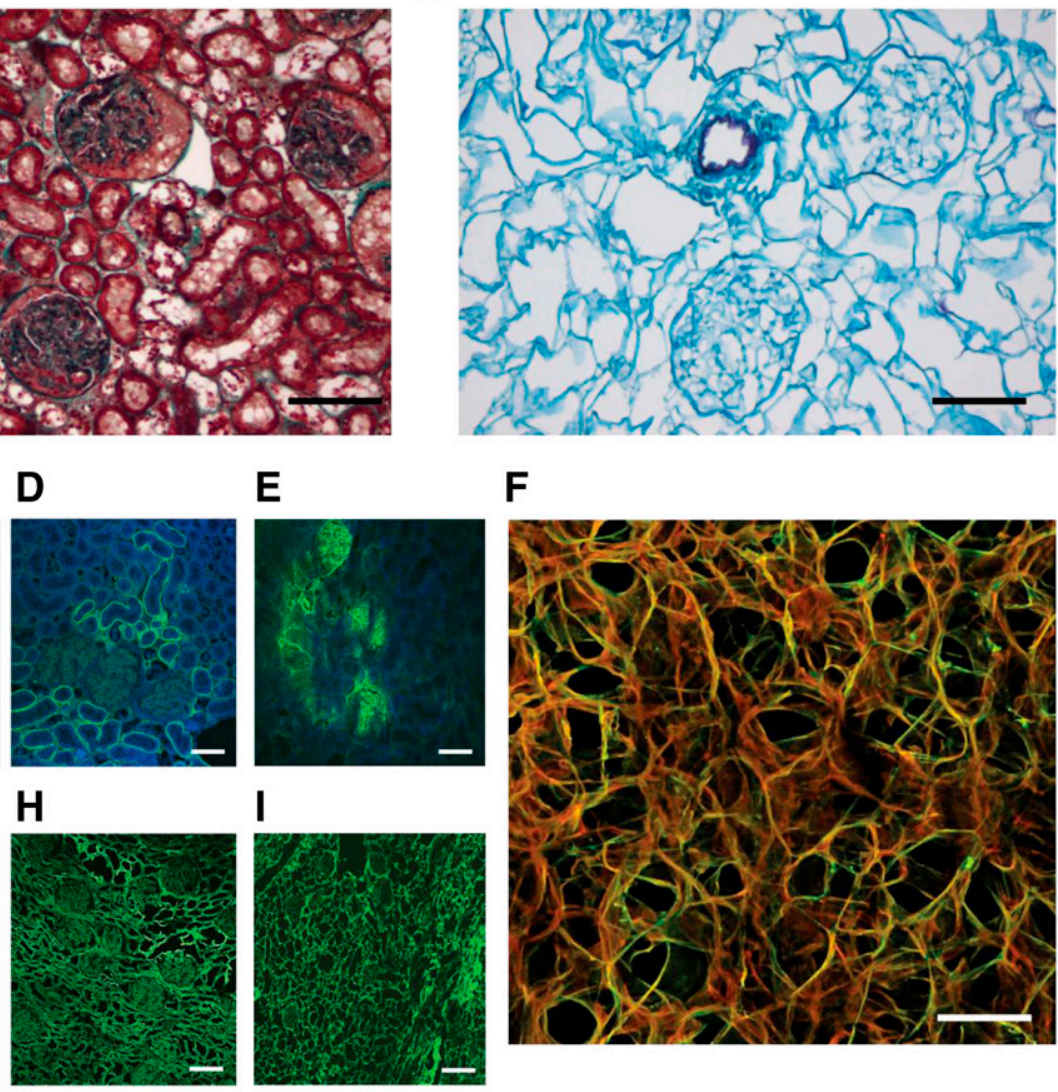

$\mathbf{F}$

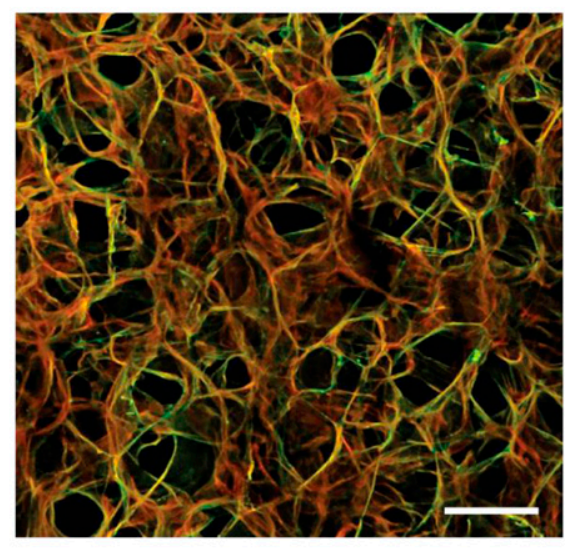

M

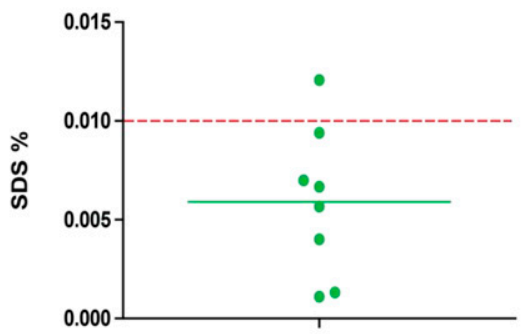

Decellularized scaffolds

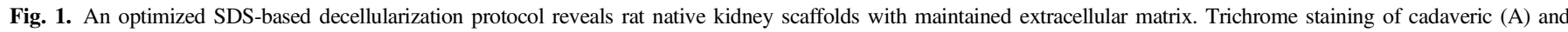

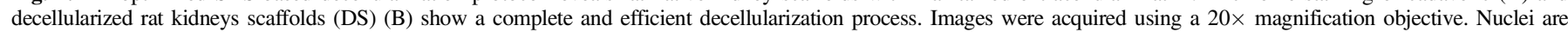

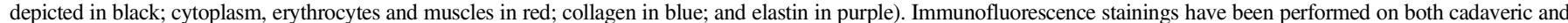

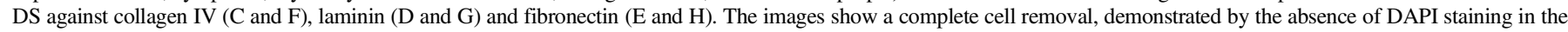

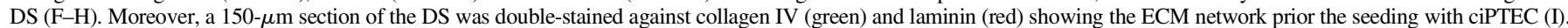

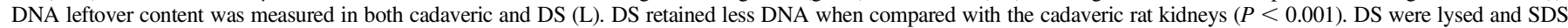

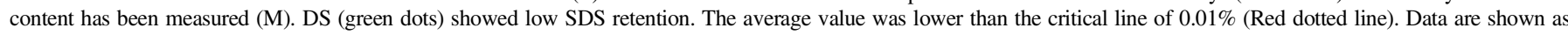

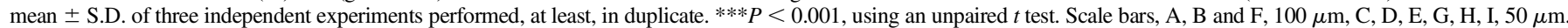

complete removal of remaining rat DNA, a procedure that lasted up to 5 days. Complete decellularization of rat kidneys was confirmed by the absence of hematoxylin staining (specific for basophilic nuclear material; Fig. 1B) in Masson trichrome staining, compared with the cadaveric kidneys (Fig. 1A). Moreover, the renal ECM, consisting mainly of collagen, was preserved. Also, the ECM microarchitecture of former tubules, glomeruli, and vessels was also retained.

Immunofluorescence of scaffolds was performed to confirm further the ECM microstructure preservation, which was demonstrated by the absence of 4',6-diamidino-2-phenylindole (DAPI) in the acellular scaffold compared with cadaveric kidneys. Moreover, strong collagen
IV and laminin signals were detected in tubular and glomerular basements (Fig. 1, H, H, F, and G). Fibronectin was more intense in the glomerulus of the cadaveric kidney (Fig. $1 \mathrm{E}$ and I) and more uniformly distributed in the DS (Fig. 1F).

DNA leftover content was determined as proof of complete decellularization, which appeared to be $0.20 \pm 0.07 \mathrm{mg}$ for the DS versus $2.60 \pm 0.21 \mathrm{mg}$ for native kidney $(P<0.0001$; Fig. $1 \mathrm{~L})$. Finally, SDS leftover content was also determined, as this can be harmful to the scaffolds and cytotoxic during the later recellularization process. In our DS, the SDS content was $0.006 \% \pm 0.003 \%(N=8$; Fig. $1 \mathrm{M})$ below the reported threshold level of $0.01 \%$ (Nakayama et al., 2013). 
A

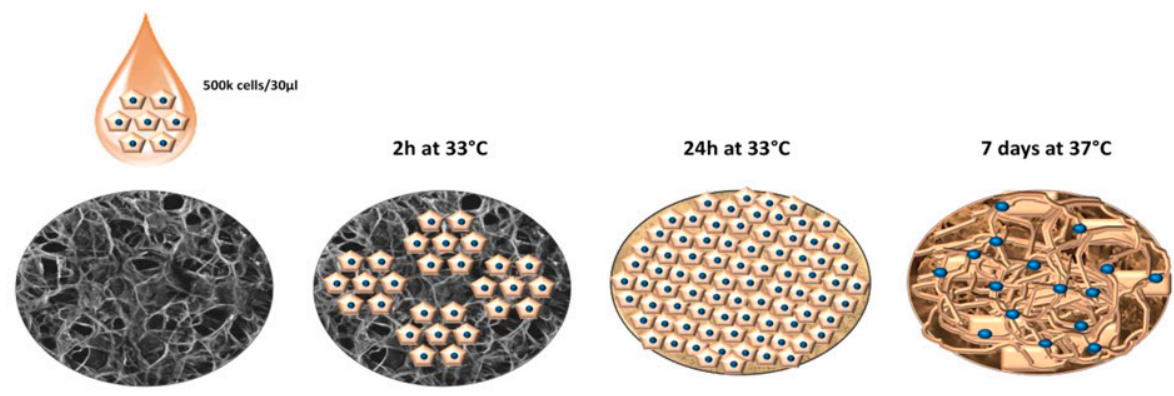

B

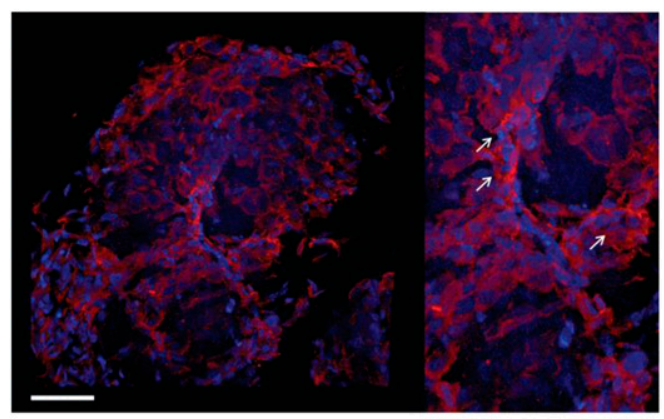

C

D

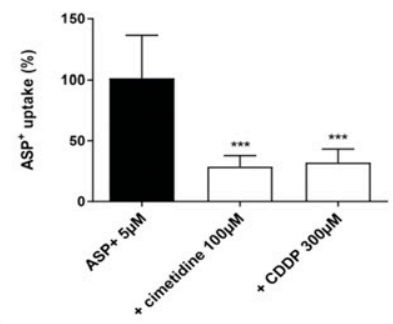

H

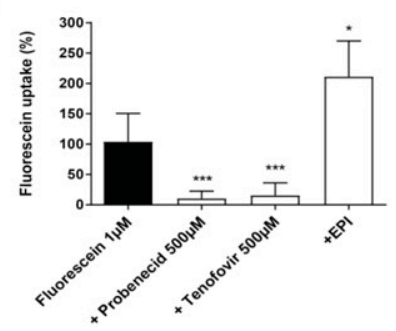

M

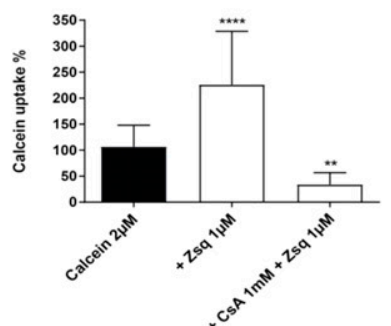

E

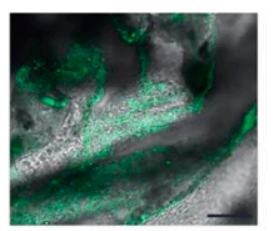

F

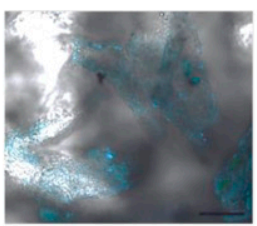

G

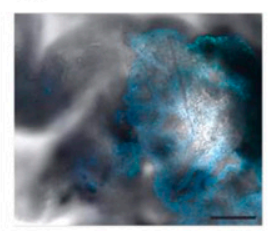

I

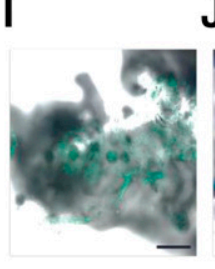

J

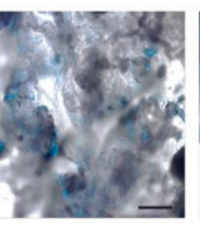

K

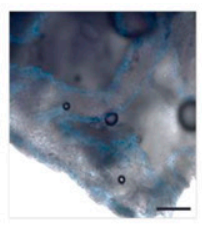

$\mathbf{L}$

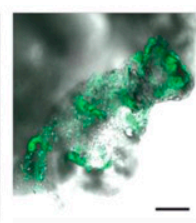

$\mathbf{N}$

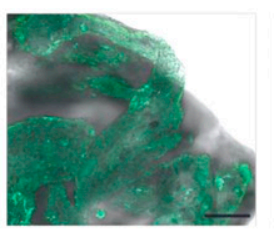

0

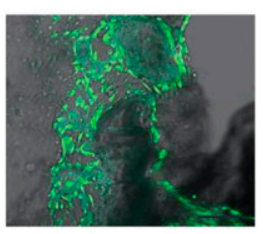

P

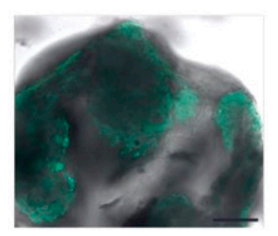

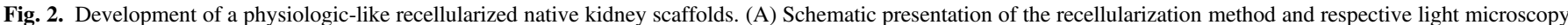

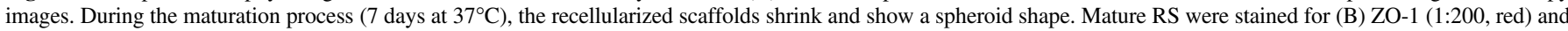

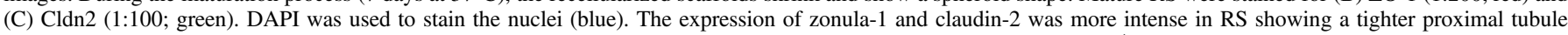

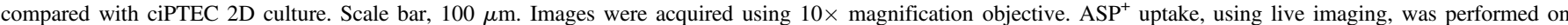

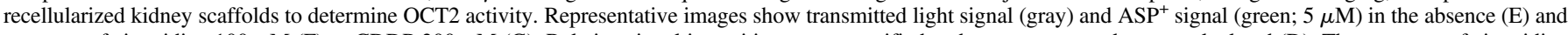

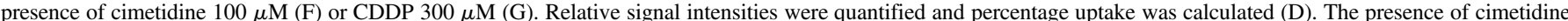

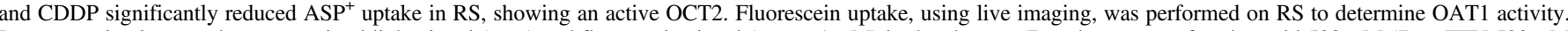

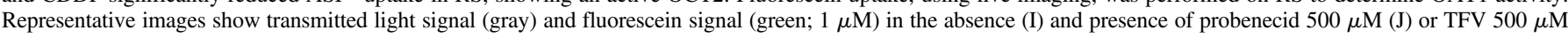


TABLE 1

Gene expression levels, in cycle threshold $\left(\mathrm{C}_{\mathrm{t}}\right)$ of ciPTEC-OAT1 in 2D cultures, in recellularized scaffolds cultures

(3D), and in human kidney (HuK)

Data are shown as mean \pm S.D. of three independent experiments performed, at least, in duplicate.

\begin{tabular}{lcccc}
\hline & & $\mathrm{C}_{\mathrm{t}}(2 \mathrm{D})$ & $\mathrm{C}_{\mathrm{t}}(3 \mathrm{D})$ & $\mathrm{C}_{\mathrm{t}}\left(\mathrm{HuK}^{a}\right)$ \\
\hline Housekeeping genes & GAPDH & $22.4 \pm 1.2$ & $22.7 \pm 0.4$ & $25.5 \pm 1.1$ \\
& HPRT1 & $23.1 \pm 0.1$ & $23.5 \pm 0.4$ & n.a. \\
Epithelial markers & ZO-1 & $24.0 \pm 0.3$ & $28.2 \pm 0.9$ & n.a. \\
& CLDN-2 & $32.7 \pm 0.8$ & $30.7 \pm 0.8$ & n.a. \\
& AQP-1 & $30.6 \pm 0.6$ & $28.5 \pm 1.2$ & n.a. \\
& OCT-2 & $33.9 \pm 4.2$ & $29.6 \pm 1.4$ & $30.3 \pm 1.8$ \\
& OAT-1 & $26.2 \pm 1.1$ & $25.0 \pm 5.1$ & $24.6 \pm 1.6$ \\
& P-gp & $30.8 \pm 1.1$ & $28.8 \pm 4.5$ & $30.3 \pm 1.8$ \\
& BCRP & $30.6 \pm 1.6$ & $29.7 \pm 1.6$ & n.a. \\
& MRP-4 & $29.4 \pm 1.1$ & $28.8 \pm 3.7$ & $29.3 \pm 1.0$ \\
& MATE-1 & $30.6 \pm 0.4$ & $25.2 \pm 1.2$ & n.a. \\
& MATE-2K & $28.8 \pm 0.5$ & $26.7 \pm 1.0$ & n.a. \\
& & $33.9 \pm 4.2$ & $29.6 \pm 1.4$ & $30.3 \pm 1.8$ \\
& GLUT-1 & $26.2 \pm 1.1$ & $25.0 \pm 5.1$ & $24.6 \pm 1.6$ \\
\end{tabular}

OATP4C1, organic anion transporter polypeptide $4 \mathrm{C} 1$

${ }^{a} \mathrm{HuK}$, human kidney, unpublished data provided by Vriend, J. et al.; n.a. (not available).

Physiologic Characterization of the Recellularized Native Kidney Scaffolds. The recellularization procedure using $150-\mu \mathrm{m}$ thick DS sections, sterilized in $0.2 \%$ (w/v) peracetic acid, consisted of four steps (Fig. 2A). First, a concentrated cell suspension was placed on the DS and incubated for 2 hours at $33^{\circ} \mathrm{C}$ to allow cells to attach to the scaffolds. Next, medium was added and the RS were kept for another 22 hours at $33^{\circ} \mathrm{C}$ to allow cell proliferation. Finally, the scaffolds were moved to $37^{\circ} \mathrm{C}$ for 7 days to allow cell maturation. During the maturation process, the RS shrank and showed a spheroid shape (Fig. 2A). For epithelial phenotypical characterization in $3 \mathrm{D}$, RS were stained for ZO-1, as a general tight epithelial marker (Kiuchi-Saishin et al., 2002), and Cldn-2, which is more specific for PTEC (Yu, 2015). Compared with ciPTEC 2D culture (Supplemental Fig. 1), the 3D RS cultures show higher expression of ZO-1 (Fig. 2B) and Cldn-2 (Fig. 2C), and formation of 3D structures (white arrows on Fig. 2, B and C), demonstrative of confluent proximal tubule formation. In addition, the mRNA expression levels demonstrated an upregulation of CLDN-2 as well, but a downregulation of ZO-1 (Table 1). Additionally, gene expression of important PTEC proteins was determined by real-time quantitative PCR. An upregulation of other epithelial markers (AQP-1) and renal transporters involved in the renal excretion of CDDP (OCT-2, MATE-1, and MATE-2K), TFV (OAT-1, MRP-4), and CsA (P-gp) was found (Table 1).

To further confirm improved physiology in RS compared with 2D cell cultures, we evaluated the functions of the relevant transporters, OCT2, OAT1, and P-gp. To determine OCT2 activity, we conducted an uptake study with $\mathrm{ASP}^{+}$as a fluorescent marker substrate (Schlatter et al., 2002; Schophuizen et al., 2013; Caetano-Pinto et al., 2016). Figure 2, D-G, demonstrates that specific uptake of $\mathrm{ASP}^{+}$was inhibitable by cimetidine, a well known OCT2 inhibitor, or by CDDP, supporting the functional presence of this transporter. Representative images confirmed a reduced intracellular $\mathrm{ASP}^{+}$accumulation in the presence of cimetidine (Fig. 2F) and CDDP (Fig. 2G), and in Supplemental Fig. 5. To determine OAT1 activity, we used fluorescein as the marker substrate (Masereeuw et al., 1994; Sun et al., 2001; Hesse et al., 2004). We tested the cellular uptake in the absence and presence of probenecid (a well known OAT1 inhibitor), TFV, and efflux pump inhibitors (EPI; MK571 to inhibit MRP-4, and KO143 to inhibit BCRP; Fig. 2H) (Caetano-Pinto et al., 2016). Representative images show a reduced intracellular fluorescein accumulation in the presence of probenecid (Fig. 2J) and TFV (Fig. 2K) compared with control. In the presence of the efflux pump inhibitors, intracellular fluorescein accumulation increased (Fig. 2L), supporting their contribution to renal fluorescein handling in RS. Large-field representative images are shown in Supplemental Fig. 6.

P-gp activity was evaluated with calcein-AM, which is effluxed by the transporter (Caetano-Pinto et al., 2016), in absence and presence of competitors zosuquidar or CsA. Upon transporter inhibition, an increased intracellular fluorescence should be observed owing to the conversion of calcein-AM into the fluorescent calcein; however, in the coexposure to Zsq and CsA less calcein was detected owing to a decreased cell viability by CsA that accumulated upon P-gp inhibition by Zsq. Representative images show an increased intracellular calcein signal in the presence of Zsq (Fig. 2O), whereas less calcein accumulation was detected after exposure to CsA together with Zsq (Fig. 2P), most probably owing to toxicity. In the presence of Zsq alone, intracellular fluorescence was increased by $2.1 \pm 1.0$-fold (Fig. $2 \mathrm{M}$ ), confirming the inhibitory potency of Zsq (King et al., 2017). Large-field representative images are shown in Supplemental Fig. 7.

Recellularized Scaffolds in Cisplatin Toxicity Evaluation. CDDP exerts a specific nephrotoxic effect because it is effectively being taken up by OCT2 (Ciarimboli et al., 2005, 2010), which is present at the basolateral membrane of the PTEC. CDDP nephrotoxicity has been widely studied in various in vitro and in vivo models (Astashkina et al., 2012a,b; Kim et al., 2014; Freedman et al., 2015; Morizane et al., 2015; Takasato et al., 2015), and therefore was selected as model drug. We examined CDDP toxicity using a PrestoBlue viability assay, as well as

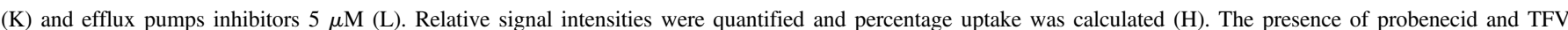

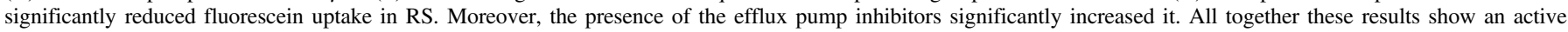

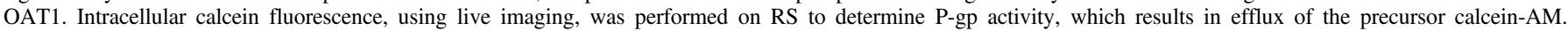

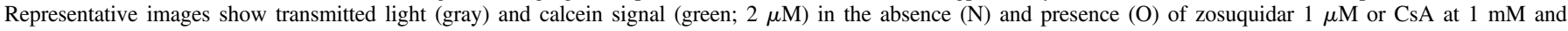

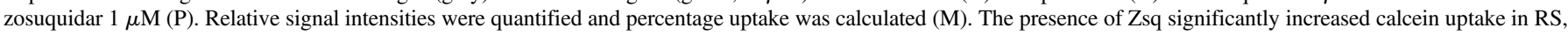

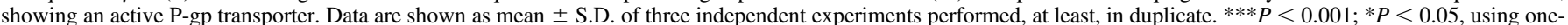
way analysis of variance analysis followed by Dunnett's multiple comparison test. White scale bar, $100 \mu \mathrm{m}$; black scale bar, $250 \mu \mathrm{m}$. 
A

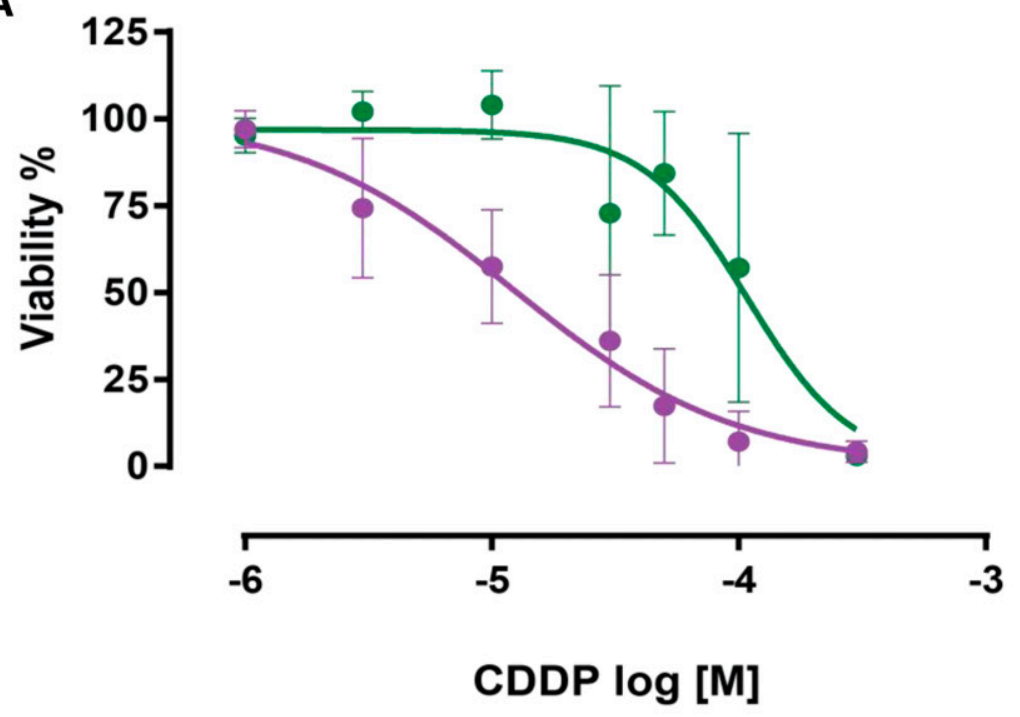

B

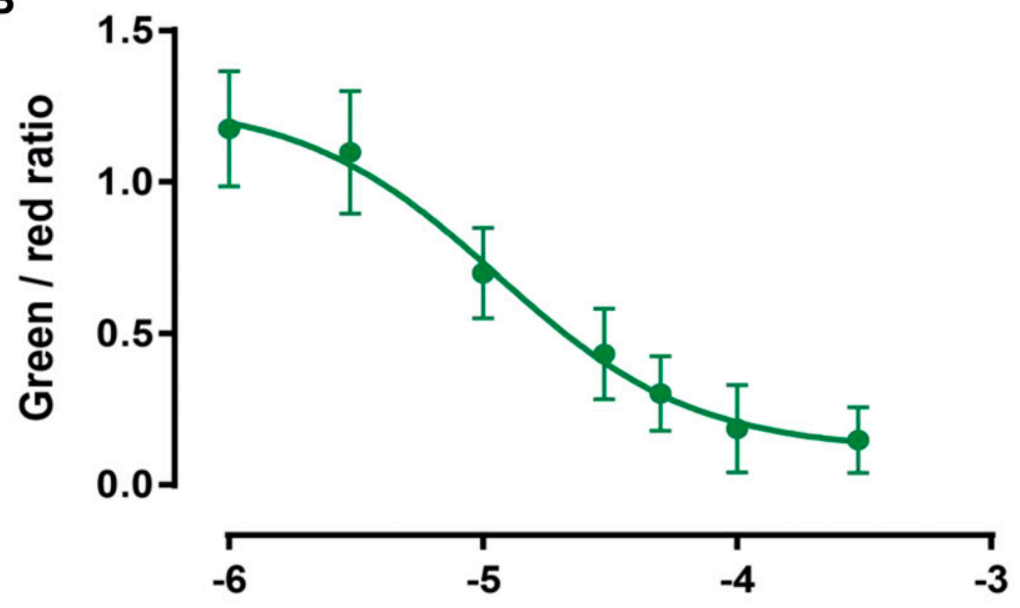

CDDP log [M]

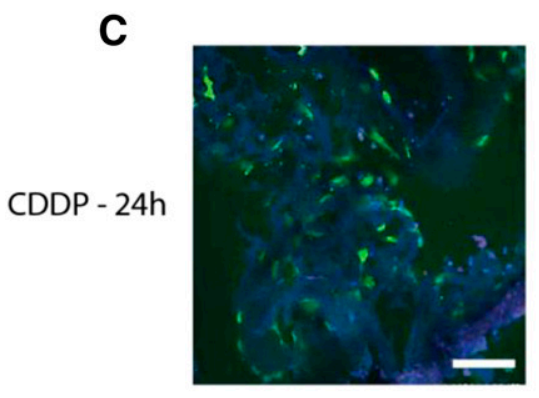

Untreated

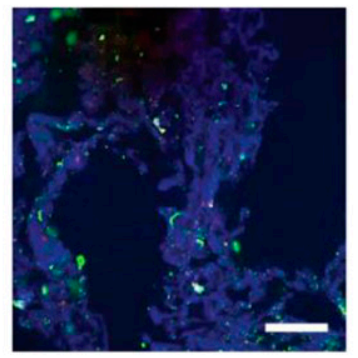

$30 \mu \mathrm{M}$

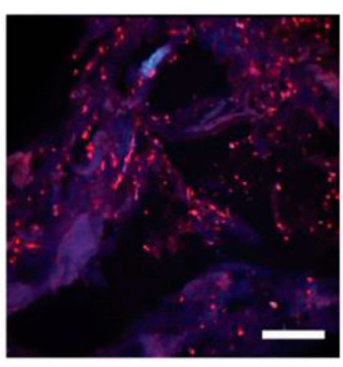

$300 \mu \mathrm{M}$
Fig. 3. Recellularized native kidney scaffolds respond to CDDP exposure. Matured ciPTECOAT1 2D and RS were treated with a concentration range of CDDP $(0-300 \mu \mathrm{M})$ for 24 hours in complete medium, followed by the PrestoBlue assay (A) or Live/Dead assay (B). The PrestoBlue assay showed that RS (purple line) seemed to be more sensitive to the drug compared with 2D ciPTEC-OAT1 cultures (green line). Live/Dead was performed in RS exposed to CDDP (green line). (C) Representative Live-Dead assay images. The number of ethidium homodimer-positive cells (red) increased with increasing concentrations of cisplatin. Nonlinear regression analysis was performed using GraphPad Prism 6.07. Data are shown as mean \pm S.D. of three independent experiments performed, at least, in duplicate. White scale bar, $100 \mu \mathrm{m}$.
Live/Dead staining assay. The PrestoBlue viability assay is based on the resazurin-resorufin reaction. Resazurin is a blue dye, itself weakly fluorescent until irreversibly reduced to the highly red fluorescent resorufin by reduction in the live-cell environment. We exposed ciPTEC $2 \mathrm{D}$ cultures and RS to a range of CDDP concentrations for 24 hours.
A clear concentration-dependent reduction in cell viability was observed for both 2D ciPTEC culture and RS (Fig. 3A; $P<0.001$ ). Analysis of the data revealed that the drug concentration was leading to a $50 \%$ reduction in cell viability, which is presented in Table 2. Overall, our novel ciPTEC 3D model (RS) demonstated higher sensitivity to CDDP (Table 2). 
TABLE 2

Cisplatin toxicity in ciPTEC in 2D cultures and recellularized scaffolds in this study in comparison with published in vitro data

\begin{tabular}{lccl}
\hline & Condition & TC $_{50}(\mu \mathrm{M})$ & \multicolumn{1}{c}{ Reference } \\
\hline PrestoBlue assay & ciPTEC-OAT1 2D $(24 \mathrm{~h})$ & $108 \pm 10$ & \\
& RS $(24 \mathrm{~h})$ & $12 \pm 5$ & \\
Live/Dead assay & RS $(24 \mathrm{~h})$ & $11.4 \pm 1.1$ & \\
Literature & ciPTEC-OAT1 2D (72 h) & $32 \pm 18$ & Caetano-Pinto et al. (2017) \\
& MDCK $(24 \mathrm{~h})$ & $18 \pm 2$ & Garbutcheon-Singh et al. (2013) \\
& HEK-293 (72 h) & $2.3 \pm 0.3$ & Shen et al. (2013) \\
HEK-293-OCT2 (72 h) & $1.4 \pm 0.2$ & \\
HEK-293-MATE1 (72 h) & $1.1 \pm 0.1$ & \\
\hline HEK-293-MATE2k (72 h) & $0.9 \pm 0.1$ & \\
\hline
\end{tabular}

HEK-293-MATE1, human embryonic kidney cells 293 overexpressing MATE1; HEK-293-MATE2, human embryonic kidney cells 293 overexpressing MATE2; HEK-293-OCT2, human embryonic kidney 293 cells overexpressing OCT-2s; MDCK, Madin-Darby canine kidney.

The basis of the Live/Dead assay is the conversion of calcein-AM into the green-fluorescent calcein after acetoxymethylester hydrolysis by intracellular enzymes, demonstrative of esterase activity in viable cells. Furthermore, the ethidium homodimer is a weakly membraneimpermeable fluorescent dye until bound to DNA and emits red fluorescence only in cells with disrupted plasma membranes. The ratio of green over red fluorescence is an indication of cell viability, which was reduced in a concentration-dependent manner after CDDP treatment (Fig. 3B; Table 2). Representative images of CDDP-treated scaffolds with this assay are presented in Fig. 3C. More details are presented in Supplemental Fig. 2.

Recellularized Scaffolds in Tenofovir Toxicity Evaluation. The nephrotoxic potential of TVF is attributable to specific uptake by OAT1 (Takeda et al., 2002; Kohler et al., 2011), present at the basolateral membrane of PTEC. This was confirmed earlier in 2D cultures of ciPTEC overexpressing OAT1 (Nieskens et al., 2016). Here, TVF demonstrated concentration-dependent reduction in viability in RS, using the PrestoBlue assay as well (Fig. 4A; $P<0.001$ ) and Live/Dead staining (Fig. 4B). Moreover, in ciPTEC 2D cultures, increasing the exposure time to 48 hours resulted in more severe effects that conform to the nature of intrinsic toxicants (Cheng et al., 2016), and RS showed similar $\mathrm{TC}_{50}$ values after 24- and 48-hour exposure. RS TFV nephrotoxicity data (Live/Dead assay) displayed similar $\mathrm{TC}_{50}$ compared with HEK-293 and Chinese hamster ovary (CHO) cell lines (Table 3). Representative images are presented in Supplemental Fig. 2.

Recellularized Scaffolds in Cyclosporine A Toxicity Evaluation. CsA was reported to be effluxed via P-gp at the apical membrane of PTEC (Anglicheau et al., 2006; Netsomboon et al., 2016), and CsA nephrotoxicity could be enhanced by blocking the transporter with PSC833 (valspodar) (Anglicheau et al., 2006). Several attempts [Anglicheau et al. (2006); Huang et al. (2015)] have been made to demonstrate CsA nephrotoxicity in PTEC in vitro, but in most studies a clear concentration-dependent effect was not found. Again, in our study, a dose-dependent reduction in cell viability in ciPTEC 2D and in $3 \mathrm{D}$ cultures (RS) exposed to CsA was observed (Fig. 5A; Table 4). The presence of Zsq increased the toxicity in both cases, supporting the contribution of P-gp in the efflux of CsA as demonstrated in Fig. 2, without showing an intrinsic toxicity at the concentrations used (Supplemental Fig. 3). The Live/Dead assay confirmed the dose-dependent reduction in cell viability of RS exposed to CsA in the absence and presence of Zsq, respectively (Fig. 5B; Table 4). Representative images of CsA-treated scaffolds demonstrate an increased number of red cells at increasing CsA concentration (Fig. 5C; Supplemental Fig. 2).

\section{Discussion}

A portion of newly developed pharmaceuticals fail during the preclinical and clinical phases as a result of nephrotoxicity (Cook et al., 2014). In the past few years, various PTEC in vitro models have been developed in two dimensions (Brown et al., 2008; Wu et al., 2009). The choice of PTEC source, however, appeared crucial, as demonstrated by Jenkinson et al. (2012), who compared primary PTEC with the cell line HK2, which is often used in nephrotoxicity studies. Their main conclusion was that HK2 is of limited value as a model for drug transporter expression and function in human kidney (Jenkinson et al., 2012). We believe that a 3D model could better mimic the in vivo situation by enhancing expression levels of the transporters involved in drug handling more closely to physiologic levels (Sanchez-Romero et al., 2016). It is rather complex to define an appropriate nephrotoxicity model. Limited available data (e.g., $\mathrm{TC}_{50}$ ), transporter expression levels, robustness, and reproducibility of the model often compromise a solid comparison. Up to now, several 2D and imaging-based high-throughput screening platforms have been established as a foundation for analyzing a wide range of compounds (Kandasamy et al., 2015; Ramm et al., 2016; Su et al., 2016; Loo and Zink, 2017). On the other hand, most of the newly developed 3D models have shown renal proximal tubule function but supply limited data on drug nephrotoxicity screening (Astashkina et al., 2012a; Davies, 2014; Homan et al., 2016; Weber et al., 2016; King et al., 2017).

We developed a 3D drug screening platform using DS recellularized with ciPTEC-OAT1, followed by a validation using three widely described nephrotoxic drugs. The 3D cell culture improved ciPTECOAT1 function and resulted in an upgraded model characterized by many key functions of proximal tubule epithelial cells, in terms of epithelial phenotype, transporter expression, and the demonstration of their function. This is probably the result of the combination of cells recapitulating major PTEC functions within the natural renal matrix. Interestingly, our 3D model showed more intense presence of tight junctions compared with 2D ciPTEC-OAT1 cultures, as demonstrated by the ZO- 1 and Cldn- 2 protein expressions. Nevertheless, the formation of $3 \mathrm{D}$ structures is heterogeneous. We believe that this is attributable to the fact that the scaffolds harbored originally $>10$ cell types and we recellularized with only one. PTEC are normally present in our nephrons as cylindrical, polarized structures with distinct basolateral (blood side) and apical (pro-urine side) membranes. RS showed active OCT2, OAT1, and P-gp functions when marker substrates were used with a defined set of inhibitors, which was confirmed with live imaging. More importantly, CDDP, TFV, and CsA could interfere with the uptake of $\mathrm{ASP}^{+}$(OCT2) and fluorescein (OAT1), and the efflux of calcein (P-gp), respectively. RS showed a clear upregulation of relevant epithelial markers 
A

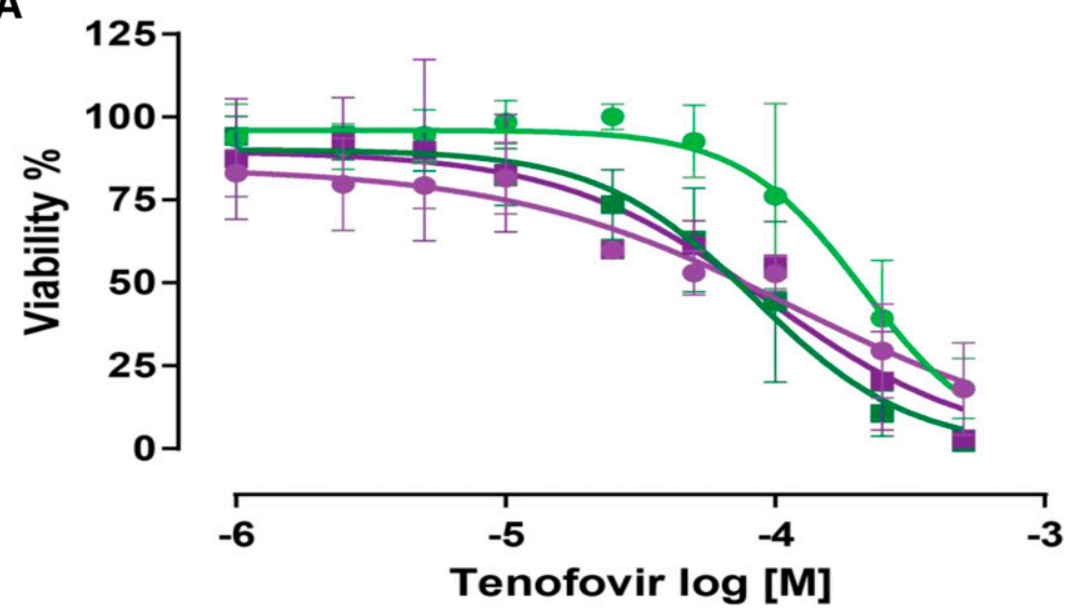

B

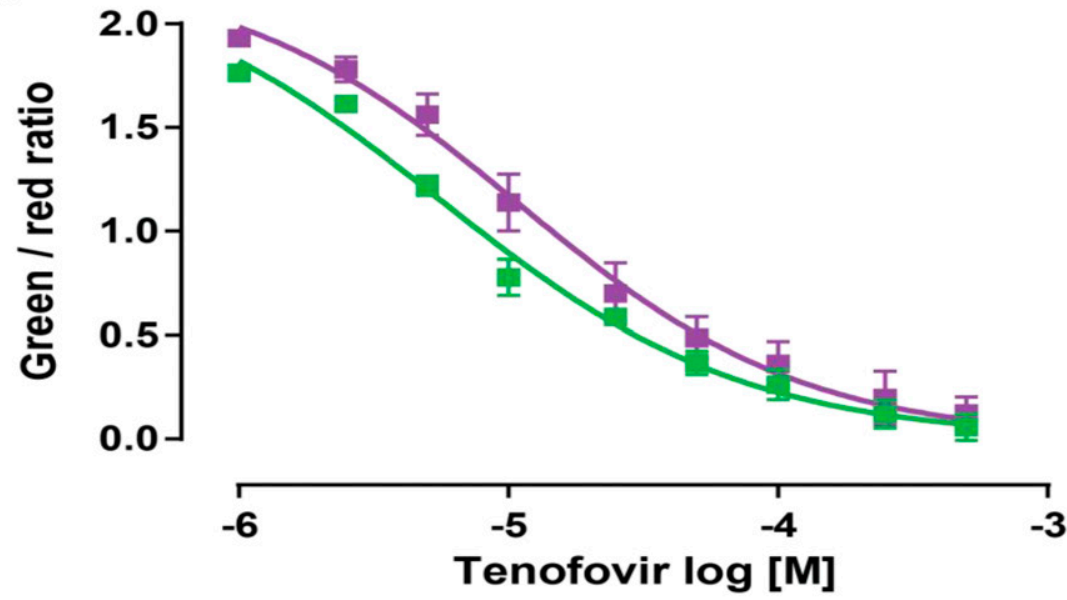

C

$3 D-24 h$
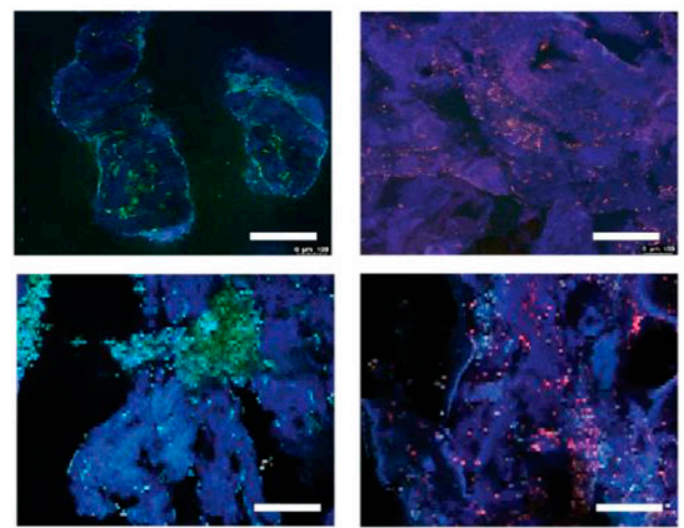

Untreated

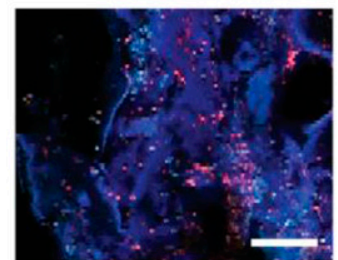

$50 \mu \mathrm{M}$
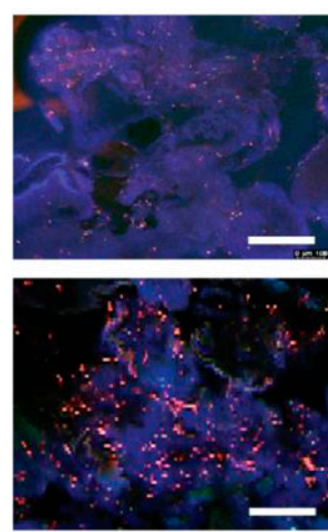

$500 \mu \mathrm{M}$
Fig. 4. Recellularized native kidney scaffolds respond to TFV exposure. Matured ciPTEC-OAT1 2D and RS were treated with a concentration range of TFV $(0$ $500 \mu \mathrm{M})$ for 24 and 48 hours, followed by the PrestoBlue assay (A) or Live/Dead assay (B). 2D ciPTEC-OAT1 and RS cultures were exposed to tenofovir for 24 hours (green and purple dot, respectively) vs. 48 hours (green and purple square, respectively). Live/Dead assay was performed in RS exposed to TFV for 24 hours (purple dot) vs. 48 hours (green square). (C) Representative Live-Dead assay images. Ethidium homodimer-positive cells number (red) increased with increasing concentrations of TFV. Nonlinear regression analysis was performed using Graphpad prism 6.07. Data are shown as mean \pm S.D. of three independent experiments performed, at least, in duplicate. ${ }^{*} P<0.05 ; * * * P<0.001$, using one-way analysis of variance analysis followed by Dunnett's multiple comparison test. White Scale bar, $100 \mu \mathrm{m}$.
(AQP-1 and CLDN-2) and all transporters relevant for nephrotoxicity screening of the drugs tested (OCT2, MATE-1 and MATE-2K, OAT1, BCRP, MRP-4, and P-gp). Finally, nephrotoxicity studies with three different well studied compounds resulted in reproducible data within a small cell passage number interval. Our previous study demonstrated, however, the stability of the expression levels over the spanning of a large passage (Nieskens et al., 2016). Future research will be designed to further characterize the RS for drug disposition studies.

CDDP nephrotoxicity was compared between 2D and 3D cell models. The two viability assays applied revealed comparable $\mathrm{TC}_{50}$ values in $3 \mathrm{D}$, 
TABLE 3

Tenofovir toxicity in ciPTEC in 2D cultures and recellularized scaffolds in this study in comparison with published in vitro data

\begin{tabular}{lccc}
\hline & Condition & TC $_{50}(\mu \mathrm{M})$ & Reference \\
\hline PrestoBlue assay & ciPTEC-OAT1 2D $(24 \mathrm{~h})$ & $212 \pm 11$ & \\
& ciPTEC-OAT1 2D $(48 \mathrm{~h})$ & $85 \pm 9$ & \\
RS $(24 \mathrm{~h})$ & $97 \pm 8$ & \\
Live/Dead assay & RS $(48 \mathrm{~h})$ & $11.0 \pm 3.0$ & Nieskens et al. (2016) \\
RS $(24 \mathrm{~h})$ & $5.5 \pm 2.0$ & \\
RS $(48 \mathrm{~h})$ & $114 \pm 25$ & Cihlar et al. (2009) \\
& ciPTEC-OAT1 2D $(24 \mathrm{~h})$ & $223 \pm 67$ & Zhang et al. (2015) \\
\hline
\end{tabular}

HEK-OAT1, human embryonic kidney cells 293 overexpressing OAT-1.

that were 10 -fold lower compared with $2 \mathrm{D}$, meaning an increased toxicity in the latter compatible with increased sensitivity. Reported $\mathrm{TC}_{50}$ values in OCT2 overexpression cell systems vary between 1 and $30 \mu \mathrm{M}$ (Table 2) and are in line with our 3D nephrotoxicity findings. The importance of OCT2 in CDDP uptake and nephrotoxicity development in PTEC has been shown in many in vitro preclinical and clinical studies (Ciarimboli et al., 2005, 2010), but not so much is known about CDDP efflux. MATE-1 and MATE-2K have been suggested in this respect, both being expressed at the brush-border membrane of PTEC (Ciarimboli et al., 2010; Ciarimboli, 2011; Sprowl et al., 2013). RS showed an increased expression of OCT2, MATE-1, and MATE-2K genes compared with ciPTEC 2D cultures; however, despite this upregulation in efflux transporters, CDDP sensitivity was not decreased. It is known that MATE- $1^{-1-}$ mice treated with CDDP showed increased nephrotoxicity and increased renal CDDP accumulation compared with wild-type animals. Less is known about the specific role of MATE-2K in CDDP efflux (Yonezawa and Inui, 2011), nor has the role of the two transporters in humans been defined (Nakamura et al., 2010; Sprowl et al., 2013). Studies have demonstrated the uptake of CDDP by human MATEs, but only by reversing the proton gradient (Tanihara et al., 2009; Sauzay et al., 2016), which is not physiologically relevant. Our data are compatible with a minor role for MATEs in CDDP efflux in ciPTEC-OAT1.

TFV-induced nephrotoxicity was recently shown using 2D cultures of ciPTEC-OAT1 and-OAT3 (Nieskens et al., 2018). In addition, TFV efflux from PTEC is mostly driven via MRP4, reducing its cytotoxic potential (Kohler et al., 2011). Here we confirmed that RS express both OAT1 and MRP4 and that both are involved in renal tubule TFV handling. The Live/Dead assay appeared to be more sensitive to TFV nephrotoxicity than the PrestoBlue assay, even though the latter should reflect the mitochondrial events associated with TFV toxicity (Boncler et al., 2014). This might be explained by the qualitative nature of the Live/Dead assay - the collection of signal intensities from confocal microscope images - whereas the PrestoBlue method quantifies the reduced metabolic capacity of cells. Also, the sensitivity in ciPTEC-OAT1 in 2D and 3D is 5- to 10-fold less compared with OAT1overexpressing CHO or HEK cells (Cihlar et al., 2009; Zhang et al., 2015; Nieskens et al., 2016), which can be explained by the absence of MRP4 in CHO or HEK cells. The balance between the influx and efflux transporter clearly affects the accumulation of TFV in the tissue. As demonstrated, when cells are cultured in 3D, the expression levels of OAT1 and MRP4 are higher than in 2D and close to human kidney cortex levels, further supporting the physiologic relevance of the $3 \mathrm{D}$ platform. Nevertheless, we cannot be entirely certain that the differences in toxicant sensitivity result from transporter expression levels solely or are inherent to the sensitivity differences between 2D and 3D platforms. To address this, we performed a pilot study with navitoclax, an antineoplastic drug used in the clinic for treatment of solid tumors. Navitoclax does not show a renal clearance profile but is handled predominantly by the liver. Our preliminary findings demonstrate a somewhat higher sensitivity in 3D compared with the 2D model but with more complete reduction in cell viability in 2D. Additional work needs to be performed to illustrate that the two systems are not appreciably different in terms of toxicant sensitivity from mechanisms that are not dependent on transport (Supplemental Fig. 8).

CsA was the third compound of interest in our nephrotoxicity screening study. CsA nephrotoxicity is being widely studied in vivo (Cheng et al., 2012; Chung et al., 2013; Lopes et al., 2014; GonzalezGuerrero et al., 2017; Gooch et al., 2017) and in the clinic (Waiser et al., 2002; Kozaki et al., 2017), but less is known about CsA nephrotoxicity effects in vitro (Anglicheau et al., 2006; Huang et al., 2015). Here, we show that CsA is actively taken up and effluxed by ciPTECs, but unlike CDDP and TFV, RS showed less sensitivity to the drug compared with the 2D model. Concerning CsA uptake into the cells, not much is known. To evaluate overt CsA toxicity, we optimized the test conditions using ciPTEC-OAT1 in terms of time (24 hours), solvent (serum-free medium), and concentration range (1-1000 $\mu \mathrm{M})$. In our 3D RS model, CsA uptake might have been lower in comparison with the 2D model, as the reduced nephrotoxicity potential cannot be explained by the changes in P-glycoprotein activity. Moreover, RS showed less sensitivity to CsA compared with other in vitro studies (Table 4), but this is probably because of the shorter incubation time [ 24 hours vs. 3 (Huang et al., 2015) and 6 days (Anglicheau et al., 2006)]. To fully understand the nephrotoxic mechanisms and possible baseline sensitivity differences between $2 \mathrm{D}$ and $3 \mathrm{D}$ platforms, future research should be directed at resolving the uptake mechanisms for CsA and fully comparing the two platforms. Overall, the most important advantage of using ciPTEC in our platform instead of overexpression cell lines such as CHO and HEK293 is the endogenous expression of multiple transporters in a cell line that also physiologically more closely resembles the renal tubular epithelium. This means, a polarized tight monolayer with distinct basolateral and apical membranes. Activity of drug transporters that are in vivo exclusively localized at the basolateral and apical membranes were shown to be functional.

Previous work demonstrated that animal experiments provide modest contributions toward human healthcare (Knight, 2008). Here, we used kidneys from surplus rats, presenting a useful way to reuse control animals from other studies. Efforts are ongoing to reduce the use of animals in toxicity screenings, for example by using a virtual resource (database) to facilitate sharing of surplus animal material developed for 
A

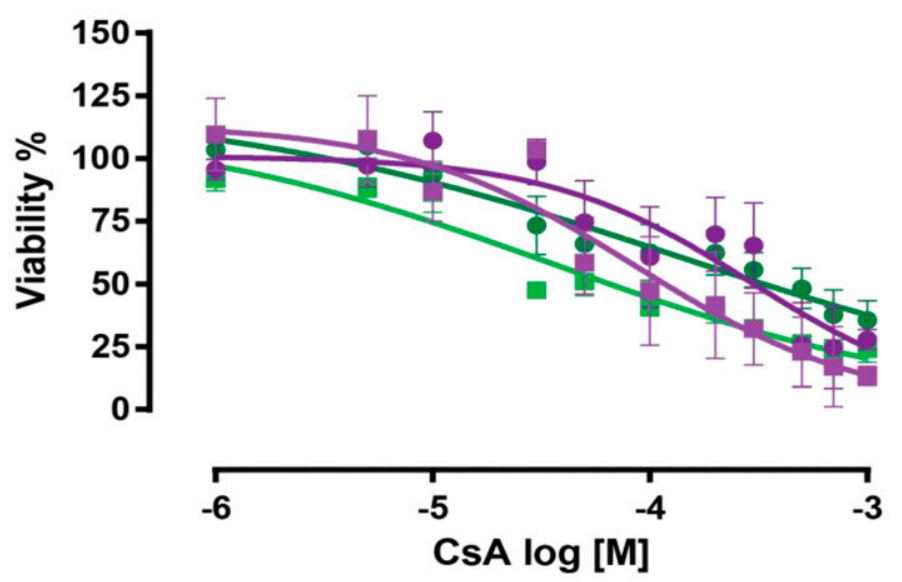

B

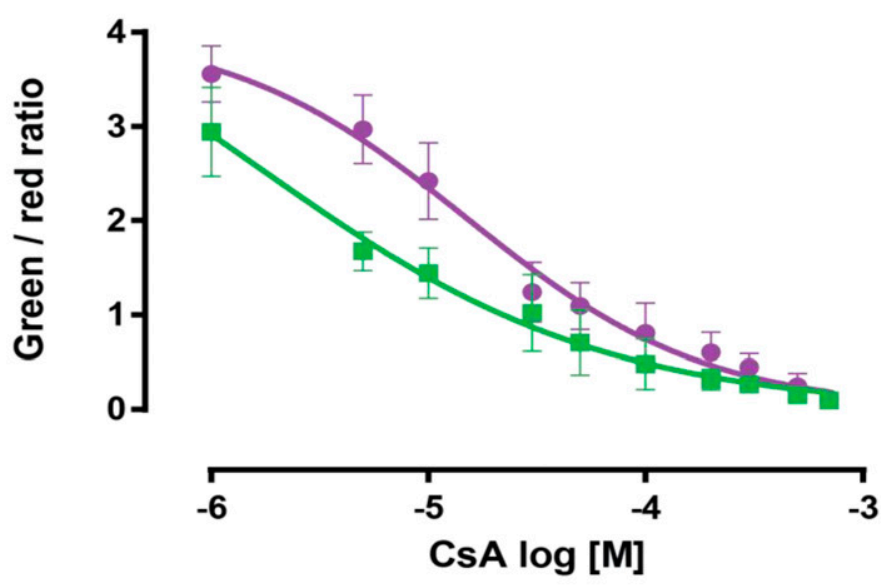

Fig. 5. Recellularized native kidney scaffolds respond to CsA exposure. Matured ciPTEC-OAT1 2D and RS were treated with a concentration range of CsA $(0-1 \mathrm{mM})$ for 24 hours, followed by the PrestoBlue assay (A) or Live/Dead assay (B). CiPTEC-OAT1 2D cultures and RS were exposed to CsA in the presence (green and purple squares, respectively) vs. absence of Zsq (green and purple dots, respectively). Live/Dead was performed in RS exposed to CsA in the presence (green square) vs. absence of Zsq (purple dot). (C) Representative live-dead assay images. Ethidium homodimer-positive cell numbers (red) increased with increasing concentrations of CsA. Nonlinear regression analysis was performed using GraphPad Prism 6.07. Data are shown as mean \pm S.D. of three independent experiments performed, at least, in duplicate. $* P<0.05 ; * * P<0.01$; $* * * P<0.001$, using one-way analysis of variance analysis followed by Dunnett's multiple comparison test. White scale bar, $100 \mu \mathrm{m}$.

C

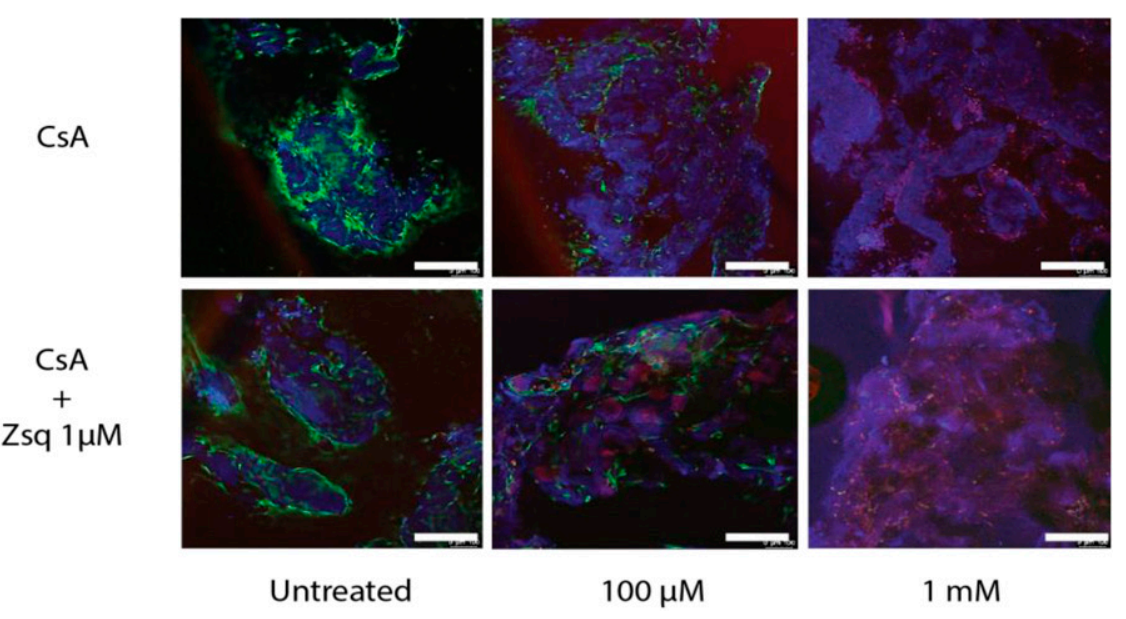

breast research by. Additionally, valid alternative models have been developed (e.g., embryonic stem cells, rodent whole embryo culture, and zebrafish) that may be useful in early preclinical safety assessments, thereby reducing human health risks and drug development costs (Brannen et al., 2016) and bypassing the use of large numbers of animals (e.g., mice and rats). As for kidney research, alternative ways following the reduction, replacement, refnement principles (3Rs) are devoted to development of engineered tissues to gain a better understanding of normal kidney development and aid the search for regenerative treatments (Gross, 2007; Davies, 2014). Still, more 
TABLE 4

Cyclosporine toxicity in ciPTEC in 2D cultures and recellularized scaffolds in this study in comparison with published in vitro data

\begin{tabular}{lccc}
\hline & Condition & TC $_{50}(\mu \mathrm{M})$ & Reference \\
\hline PrestoBlue assay & ciPTEC-OAT1 2D & $129 \pm 12$ & \\
& ciPTEC-OAT1 2D $(+$ Zsq $1 \mu \mathrm{M})$ & $35 \pm 6$ & \\
RS & $294 \pm 13$ & \\
Live/dead assay & RS (+Zsq $1 \mu \mathrm{M})$ & $87 \pm 10$ & \\
RS & $15.0 \pm 1.2$ & \\
Literature & RS $(+Z s q 1 \mu \mathrm{M})$ & $1.6 \pm 0.4$ & Huang et al. (2015) \\
& HK-2 $(72 \mathrm{~h})$ & $>50$ & \\
hPTECs $(72 \mathrm{~h})$ & $>50$ & Anglicheau et al. (2006) \\
& HRECs (CsA only; 144 h) & $7.4 \pm 0.5$ & \\
\hline
\end{tabular}

HK-2, human kidney cells 2; hPTECs, human proximal tubule epithelial cells; hRECs, human renal epithelial cells.

investments have to be made in developing of high-throughput in vitro models that mimic the human kidney and improve the prediction of drug-induced nephrotoxicity, as well as reduce use of animals in research (McGuinness, 2014).

To further improve our 3D model, a dynamic cell culture could be implemented, as shear-stress previously demonstrated to be another factor improving tissue performance in vitro. A continuous low shear stress may induce cell polarization and barrier formation (Jang et al., 2013; Weber et al., 2016), as well as increase CDDP sensitivity (Jang et al., 2013). Related to this, the kidney-on-a-chip technology is rapidly evolving for (high-throughput) nephrotoxicity drug screening and to improve preclinical drug discovery and development (Bhatia and Ingber, 2014; Polini et al., 2014; Nieskens and Wilmer, 2016; Wilmer et al., 2016). Moreover, it would also be interesting to use our newly developed platform to perform chronic exposures (e.g., daily drug exposure for 2-3 weeks), to mimic chronic injury development (DesRochers et al., 2013). Finally, the in vivo translation of in vitro obtained data are an essential element in nephrotoxicity screenings (in vitro-in vivo extrapolation). Predicting drug disposition from in vitro models is very complex, and to bypass this issue, in silico models are being developed (Jamei et al., 2009; Chen et al., 2012). These models rely on robust experimental data. Future studies should be directed at revealing the true predictive value of our platform in toxicity studies, including translatability with clinically relevant endpoints.

In conclusion, we developed a $3 \mathrm{D}$ drug screening platform by combining decellularized kidney scaffolds with ciPTEC-OAT1. Our data demonstrate that recellularized kidney scaffolds are an epithelial phenotype with PTEC characteristics and sensitivity to three known nephrotoxicants. The platform has potential application in the screening of new drugs prior to initiating clinical trials, thereby improving drug development processes.

\section{Acknowledgments}

We thank Jos Brits from the Brain Center Rudolf Magnus (UMC, Utrecht, The Netherlands) for providing us the rats; and Vivienne Verweij and Jeanne Pertijs from Radboud university medical center (Nijmegen, The Netherlands) for helping with the optimization of the decellularization protocol; and Jelle Vriend (Radboud university medical center) for providing the human kidney cortex gene expression data.

\section{Authorship Contributions}

Participated in research design: Fedecostante, Sanchez Romero, Baptista, Wilmer, Hoenderop, Masereeuw.

Conducted experiments: Fedecostante, Buono, Kerkering, Westphal.

Contributed new reagents or analytic tools: Fedecostante, Kerkering, Westphal.

Performed data analysis: Fedecostante, Buono, Kerkering, Masereeuw.
Wrote or contributed to the writing of the manuscript: Fedecostante, Sanchez Romero, Baptista, Wilmer, Hoenderop, Masereeuw.

\section{References}

Anglicheau D, Pallet N, Rabant M, Marquet P, Cassinat B, Méria P, Beaune P, Legendre C, and Thervet E (2006) Role of P-glycoprotein in cyclosporine cytotoxicity in the cyclosporinesirolimus interaction. Kidney Int 70:1019-1025.

Astashkina AI, Mann BK, Prestwich GD, and Grainger DW (2012a) A 3-D organoid kidney culture model engineered for high-throughput nephrotoxicity assays. Biomaterials 33: 4700-4711.

Astashkina AI, Mann BK, Prestwich GD, and Grainger DW (2012b) Comparing predictive drug nephrotoxicity biomarkers in kidney 3-D primary organoid culture and immortalized cell lines. Biomaterials 33:4712-4721.

Awdishu L and Mehta RL (2017) The 6R's of drug induced nephrotoxicity. BMC Nephrol 18: 124.

Bhatia SN and Ingber DE (2014) Microfluidic organs-on-chips. Nat Biotechnol 32:760-772.

Boncler M, Różalski M, Krajewska U, Podsędek A, and Watala C (2014) Comparison of PrestoBlue and MTT assays of cellular viability in the assessment of anti-proliferative effects of plant extracts on human endothelial cells. J Pharmacol Toxicol Methods 69:9-16.

Brannen KC, Chapin RE, Jacobs AC, and Green ML (2016) Alternative models of developmental and reproductive toxicity in pharmaceutical risk assessment and the 3Rs. ILAR J 57:144-156. Brown CD, Sayer R, Windass AS, Haslam IS, De Broe ME, D'Haese PC, and Verhulst A (2008) Characterisation of human tubular cell monolayers as a model of proximal tubular xenobiotic handling. Toxicol Appl Pharmacol 233:428-438.

Caetano-Pinto P, Jamalpoor A, Ham J, Goumenou A, Mommersteeg M, Pijnenburg D, Ruijtenbeek R, Sanchez-Romero N, van Zelst B, Heil SG, et al. (2017) Cetuximab prevents methotrexateinduced cytotoxicity in vitro through epidermal growth factor dependent regulation of renal drug transporters. Mol Pharm 14:2147-2157.

Caetano-Pinto P, Janssen MJ, Gijzen L, Verscheijden L, Wilmer MJ, and Masereeuw R (2016) Fluorescence-based transport assays revisited in a human renal proximal tubule cell line. Mol Pharm 13:933-944.

Chen Y, Jin JY, Mukadam S, Malhi V, and Kenny JR (2012) Application of IVIVE and PBPK modeling in prospective prediction of clinical pharmacokinetics: strategy and approach during the drug discovery phase with four case studies. Biopharm Drug Dispos 33:85-98.

Cheng Y, El-Kattan A, Zhang Y, Ray AS, and Lai Y (2016) Involvement of drug transporters in organ toxicity: the fundamental basis of drug discovery and development. Chem Res Toxicol 29: $545-563$.

Cheng CH, Shu KH, Chang HR, and Chou MC (2012) Cyclosporine-induced tubular vacuolization: the role of Bip/Grp78. Nephron, Exp Nephrol 122:1-12.

Chung BH, Lim SW, Doh KC, Piao SG, Heo SB, and Yang CW (2013) Human adipose tissue derived mesenchymal stem cells aggravate chronic cyclosporin nephrotoxicity by the induction of oxidative stress. PLoS One 8:e59693.

Ciarimboli G (2011) Role of organic cation transporters in drug-induced toxicity. Expert Opin Drug Metab Toxicol 7:159-174.

Ciarimboli G, Deuster D, Knief A, Sperling M, Holtkamp M, Edemir B, Pavenstädt H, LanversKaminsky C, am Zehnhoff-Dinnesen A, Schinkel AH, et al. (2010) Organic cation transporter 2 mediates cisplatin-induced oto- and nephrotoxicity and is a target for protective interventions. Am J Pathol 176:1169-1180.

Ciarimboli G, Ludwig T, Lang D, Pavenstädt H, Koepsell H, Piechota HJ, Haier J, Jaehde U, Zisowsky J, and Schlatter E (2005) Cisplatin nephrotoxicity is critically mediated via the human organic cation transporter 2. Am J Pathol 167:1477-1484.

Cihlar T, Laflamme G, Fisher R, Carey AC, Vela JE, Mackman R, and Ray AS (2009) Novel nucleotide human immunodeficiency virus reverse transcriptase inhibitor GS-9148 with a low nephrotoxic potential: characterization of renal transport and accumulation. Antimicrob Agents Chemother 53:150-156.

Cook D, Brown D, Alexander R, March R, Morgan P, Satterthwaite G, and Pangalos MN (2014) Lessons learned from the fate of AstraZeneca's drug pipeline: a five-dimensional framework. Nat Rev Drug Discov 13:419-431.

Davies J (2014) Engineered renal tissue as a potential platform for pharmacokinetic and nephrotoxicity testing. Drug Discov Today 19:725-729.

DesRochers TM, Suter L, Roth A, and Kaplan DL (2013) Bioengineered 3D human kidney tissue, a platform for the determination of nephrotoxicity. PLoS One 8:e59219.

Fedecostante M, Onciu OG, Westphal KGC, and Masereeuw R (2017) Towards a bioengineered kidney: recellularization strategies for decellularized native kidney scaffolds. Int J Artif Organs 40:150-158. 
Freedman BS, Brooks CR, Lam AQ, Fu H, Morizane R, Agrawal V, Saad AF, Li MK, Hughes MR, Werff RV, et al. (2015) Modelling kidney disease with CRISPR-mutant kidney organoids derived from human pluripotent epiblast spheroids. Nat Commun 6:8715.

Garbutcheon-Singh KB, Myers S, Harper BW, Ng NS, Dong Q, Xie C, and Aldrich-Wright JR (2013) The effects of 56MESS on mitochondrial and cytoskeletal proteins and the cell cycle in MDCK cells. Metallomics 5:1061-1067.

González-Guerrero C, Cannata-Ortiz P, Guerri C, Egido J, Ortiz A, and Ramos AM (2017) TLR4-mediated inflammation is a key pathogenic event leading to kidney damage and fibrosis in cyclosporine nephrotoxicity. Arch Toxicol 91:1925-1939.

Gooch JL, King C, Francis CE, Garcia PS, and Bai Y (2017) Cyclosporine A alters expression of renal microRNAs: new insights into calcineurin inhibitor nephrotoxicity. PLoS One 12 e0175242.

Gross M (2007) Cell searching. Curr Biol 17:R858-R859.

Hesse D, Sauvant C, Holzinger H, and Gekle M (2004) Apical expression or expression in a non polarized cell of hOAT1 inverses regulation by epidermal growth factor (EGF) as compared to basolateral hOAT1. Cell Physiol Biochem 14:177-186.

Homan KA, Kolesky DB, Skylar-Scott MA, Herrmann J, Obuobi H, Moisan A, and Lewis JA (2016) Bioprinting of 3D convoluted renal proximal tubules on perfusable chips. Sci Rep 6 34845.

Huang JX, Kaeslin G, Ranall MV, Blaskovich MA, Becker B, Butler MS, Little MH, Lash LH, and Cooper MA (2015) Evaluation of biomarkers for in vitro prediction of drug-induced nephrotoxicity: comparison of HK-2, immortalized human proximal tubule epithelial, and primary cultures of human proximal tubular cells. Pharmacol Res Perspect 3: 00148 .

Jamei M, Marciniak S, Feng K, Barnett A, Tucker G, and Rostami-Hodjegan A (2009) The Simcyp population-based ADME simulator. Expert Opin Drug Metab Toxicol 5:211-223.

Jang KJ, Mehr AP, Hamilton GA, McPartlin LA, Chung S, Suh KY, and Ingber DE (2013) Human kidney proximal tubule-on-a-chip for drug transport and nephrotoxicity assessment. Integr Biol 5:1119-1129.

Jansen J, Schophuizen CM, Wilmer MJ, Lahham SH, Mutsaers HA, Wetzels JF, Bank RA, van den Heuvel LP, Hoenderop JG, and Masereeuw R (2014) A morphological and functional comparison of proximal tubule cell lines established from human urine and kidney tissue. Exp Cell Res 323:87-99.

Jenkinson SE, Chung GW, van Loon E, Bakar NS, Dalzell AM, and Brown CD (2012) The limitations of renal epithelial cell line $\mathrm{HK}-2$ as a model of drug transporter expression and function in the proximal tubule. Pflugers Arch 464:601-611.

Kandasamy K, Chuah JK, Su R, Huang P, Eng KG, Xiong S, Li Y, Chia CS, Loo LH, and Zink D (2015) Prediction of drug-induced nephrotoxicity and injury mechanisms with human induced pluripotent stem cell-derived cells and machine learning methods. Sci Rep 5:12337.

Kim SY, Sohn SJ, Won AJ, Kim HS, and Moon A (2014) Identification of noninvasive biomarker for nephrotoxicity using HK-2 human kidney epithelial cells. Toxicol Sci 140:247-258.

King SM, Higgins JW, Nino CR, Smith TR, Paffenroth EH, Fairbairn CE, Docuyanan A, Shah VD, Chen AE, Presnell SC, et al. (2017) 3D proximal tubule tissues recapitulate key aspects of rena physiology to enable nephrotoxicity testing. Front Physiol 8:123.

Kiuchi-Saishin Y, Gotoh S, Furuse M, Takasuga A, Tano Y, and Tsukita S (2002) Differential expression patterns of claudins, tight junction membrane proteins, in mouse nephron segments J Am Soc Nephrol 13:875-886.

Knight A (2008) Systematic reviews of animal experiments demonstrate poor contributions toward human healthcare. Rev Recent Clin Trials 3:89-96.

Kohler JJ, Hosseini SH, Green E, Abuin A, Ludaway T, Russ R, Santoianni R, and Lewis W (2011) Tenofovir renal proximal tubular toxicity is regulated by OAT1 and MRP4 transporters Lab Invest 91:852-858.

Kozaki K, Tani K, and Yuzawa K (2017) Cyclosporine-induced hemolytic uremic syndrome following ABO incompatible living donor renal transplantation-case report. J Clin Exp Nephrol 1:28.

Liu HC, Goldenberg A, Chen Y, Lun C, Wu W, Bush KT, Balac N, Rodriguez P, Abagyan R, and Nigam SK (2016) Molecular properties of drugs interacting with SLC22 transporters OAT1, OAT3, OCT1, and OCT2: a machine-learning approach. $J$ Pharmacol Exp Ther 359-215-229.

Loo LH and Zink D (2017) High-throughput prediction of nephrotoxicity in humans. Altern Lab Anim 45:241-252.

Lopes PC, Fuhrmann A, Carvalho F, Sereno J, Santos MR, Pereira MJ, Eriksson JW, Reis F, and Carvalho $\mathrm{E}$ (2014) Cyclosporine A enhances gluconeogenesis while sirolimus impairs insulin signaling in peripheral tissues after 3 weeks of treatment. Biochem Pharmacol 91:61-73.

Masereeuw R, van den Bergh EJ, Bindels RJ, and Russel FG (1994) Characterization of fluorescein transport in isolated proximal tubular cells of the rat: evidence for mitochondrial accumulation J Pharmacol Exp Ther 269:1261-1267.

McGuinness L (2014) CRACK IT Challenge Winners Awarded $£ 4.9$ Million to Further Their Research London, National Centre for the Replacement, Refinement and Reduction of Animals in Research, London, UK

Morizane R, Lam AQ, Freedman BS, Kishi S, Valerius MT, and Bonventre JV (2015) Nephron organoids derived from human pluripotent stem cells model kidney development and injury. $\mathrm{Nat}$ Biotechnol 33:1193-1200.

Nakamura T, Yonezawa A, Hashimoto S, Katsura T, and Inui K (2010) Disruption of multidrug and toxin extrusion MATE1 potentiates cisplatin-induced nephrotoxicity. Biochem Pharmaco 80:1762-1767.

Nakayama KH, Lee CC, Batchelder CA, and Tarantal AF (2013) Tissue specificity of decellularized rhesus monkey kidney and lung scaffolds. PLoS One 8:e64134.

Netsomboon K, Laffleur F, Suchaoin W, and Bernkop-Schnürch A (2016) Novel in vitro transport method for screening the reversibility of P-glycoprotein inhibitors. Eur J Pharm Biopharm 100: $9-14$

Nieskens TTG, Peters JGP, Dabaghie D, Korte D, Jansen K, Van Asbeck AH, Tavraz NN, Friedrich T, Russel FGM, Masereeuw R, et al. (2018) Expression of organic anion transporter
1 or 3 in human kidney proximal tubule cells reduces cisplatin sensitivity. Drug Metab Dispos 46:592-599.

Nieskens TT, Peters JG, Schreurs MJ, Smits N, Woestenenk R, Jansen K, van der Made TK, Röring M, Hilgendorf C, Wilmer MJ, et al. (2016) A human renal proximal tubule cell line with stable organic anion transporter 1 and 3 expression predictive for antiviral-induced toxicity AAPS J 18:465-475.

Nieskens TT and Wilmer MJ (2016) Kidney-on-a-chip technology for renal proximal tubule tissue reconstruction. Eur J Pharmacol 790:46-56.

Nigam SK (2015) What do drug transporters really do? Nat Rev Drug Discov 14:29-44.

Peloso A, Tamburrini R, Edgar L, Wilm B, Katari R, Perin L, Murray P, and Orlando G (2016) Extracellular matrix scaffolds as a platform for kidney regeneration. Eur J Pharmacol 790: 21-27.

Polini A, Prodanov L, Bhise NS, Manoharan V, Dokmeci MR, and Khademhosseini A (2014) Organs-on-a-chip: a new tool for drug discovery. Expert Opin Drug Discov 9:335-352.

Ramm S, Adler M, and Vaidya VS (2016) A high-throughput screening assay to identify kidney toxic compounds. Curr Protoc Toxicol 69:9.10.1-9.10.26.

Sánchez-Romero N, Schophuizen CM, Giménez I, and Masereeuw R (2016) In vitro systems to study nephropharmacology: 2D versus 3D models. Eur J Pharmacol 790:36-45.

Sauzay C, White-Koning M, Hennebelle I, Deluche T, Delmas C, Imbs DC, Chatelut E, and Thomas F (2016) Inhibition of OCT2, MATE1 and MATE2-K as a possible mechanism of drug interaction between pazopanib and cisplatin. Pharmacol Res 110:89-95.

Schlatter E, Mönnich V, Cetinkaya I, Mehrens T, Ciarimboli G, Hirsch JR, Popp C, and Koepsell H (2002) The organic cation transporters rOCT1 and hOCT2 are inhibited by cGMP. J Membr Biol 189:237-244.

Schophuizen CM, Wilmer MJ, Jansen J, Gustavsson L, Hilgendorf C, Hoenderop JG, van den Heuvel LP, and Masereeuw R (2013) Cationic uremic toxins affect human renal proximal tubule cell functioning through interaction with the organic cation transporter. Pflugers Arch $\mathbf{4 6 5}$ 1701-1714.

Shen H, Yang Z, Zhao W, Zhang Y, and Rodrigues AD (2013) Assessment of vandetanib as an inhibitor of various human renal transporters: inhibition of multidrug and toxin extrusion as a possible mechanism leading to decreased cisplatin and creatinine clearance. Drug Metab Dispos 41:2095-2103.

Sprowl JA, Ness RA, and Sparreboom A (2013) Polymorphic transporters and platinum pharmacodynamics. Drug Metab Pharmacokinet 28:19-27.

Su R, Xiong S, Zink D, and Loo LH (2016) High-throughput imaging-based nephrotoxicity prediction for xenobiotics with diverse chemical structures. Arch Toxicol 90:2793-2808.

Sun W, Wu RR, van Poelje PD, and Erion MD (2001) Isolation of a family of organic anion transporters from human liver and kidney. Biochem Biophys Res Commun 283:417-422.

Takasato M, Er PX, Chiu HS, Maier B, Baillie GJ, Ferguson C, Parton RG, Wolvetang EJ, Roost MS, Chuva de Sousa Lopes SM, et al. (2015) Kidney organoids from human iPS cells contain multiple lineages and model human nephrogenesis. Nature 526:564-568.

Takeda M, Khamdang S, Narikawa S, Kimura H, Kobayashi Y, Yamamoto T, Cha SH, Sekine T, and Endou H (2002) Human organic anion transporters and human organic cation transporters mediate renal antiviral transport. J Pharmacol Exp Ther 300:918-924.

Tanihara Y, Masuda S, Katsura T, and Inui K (2009) Protective effect of concomitant administration of imatinib on cisplatin-induced nephrotoxicity focusing on renal organic cation transporter OCT2. Biochem Pharmacol 78:1263-1271.

(Treweek, J.B. et al. 2015) Whole-body tissue stabilization and selective extractions via tissuehydrogel hybrids for high-resolution intact circuit mapping and phenotyping. Nature Protocols 10: $1860-1896$

Waiser J, Slowinski T, Brinker-Paschke A, Budde K, Schreiber M, Böhler T, Hauser I, and Neumayer HH (2002) Impact of the variability of cyclosporin A trough levels on long-term renal allograft function. Nephrol Dial Transplant 17:1310-1317.

Weber EJ, Chapron A, Chapron BD, Voellinger JL, Lidberg KA, Yeung CK, Wang Z, Yamaura Y, Hailey DW, Neumann T, et al. (2016) Development of a microphysiological model of human kidney proximal tubule function. Kidney Int 90:627-637.

Wilmer MJ, Ng CP, Lanz HL, Vulto P, Suter-Dick L, and Masereeuw R (2016) Kidney-on-a-chip technology for drug-induced nephrotoxicity screening. Trends Biotechnol 34:156-170.

Wilmer MJ, Saleem MA, Masereeuw R, Ni L, van der Velden TJ, Russel FG, Mathieson PW, Monnens LA, van den Heuvel LP, and Levtchenko EN (2010) Novel conditionally immortalized human proximal tubule cell line expressing functional influx and efflux transporters. Cell Tissue Res 339:449-457.

Wu Y, Connors D, Barber L, Jayachandra S, Hanumegowda UM, and Adams SP (2009) Multiplexed assay panel of cytotoxicity in HK-2 cells for detection of renal proximal tubule injury potential of compounds. Toxicol In Vitro 23:1170-1178.

Yonezawa A and Inui K (2011) Organic cation transporter OCT/SLC22A and H(+)/organic cation antiporter MATE/SLC47A are key molecules for nephrotoxicity of platinum agents. Biochem Pharmacol 81:563-568.

Yu AS (2015) Claudins and the kidney. J Am Soc Nephrol 26:11-19.

Yu YL, Shao YK, Ding YQ, Lin KZ, Chen B, Zhang HZ, Zhao LN, Wang ZB, Zhang JS, Tang ML, et al. (2014) Decellularized kidney scaffold-mediated renal regeneration. Biomaterials 35: $6822-6828$.

Zhang X, Wang R, Piotrowski M, Zhang H, and Leach KL (2015) Intracellular concentrations determine the cytotoxicity of adefovir, cidofovir and tenofovir. Toxicol In Vitro 29:251-258.

Address correspondence to: Dr. Rosalinde Masereeuw, Utrecht University, Div. Pharmacology, Department of Pharmaceutical Sciences, Universiteitsweg 99, 3584 CG Utrecht, The Netherlands. E-mail: r.masereeuw@uu.nl 